\title{
Nivel de alfabetización estadística del alumnado universitario de letras: El caso de la Facultad de Educación de la Universidad Complutense de Madrid
}

\section{Statistical literacy level of arts college students: The case of the School of Education of the Complutense University of Madrid}

\author{
Joseph MAFOKOZI \\ Universidad Complutense de Madrid
}

Recibido: Octubre 2010

Aceptado: Mayo 2011

\section{Resumen}

En este estudio se analizan aspectos relacionados con la enseñanza/aprendizaje de las matemáticas y más específicamente con la estadística. El interés por modernizar esta materia a lo largo de los últimos 70 años se evidencia a través de numerosos reajustes legislativos habidos hasta la fecha. Ahora bien, ni estos cambios ni la apertura a la influencia de corrientes pedagógicas internacionales han conseguido producir mejoras notables en los niveles educativos secundario y universitario. Los resultados de este estudio revelan una situación compleja en la que las variables de contexto, entrada y proceso estudiadas parecen tener un efecto más bien residual sobre el rendimiento académico, especialmente en la universidad; aparentemente son otras las variables que podrían explicar mejor el rendimiento académico en este contexto.

Palabras clave: Matemáticas, Estadística, Alfabetización estadística, Legislación educativa, Actitudes, Rendimiento académico.

\begin{abstract}
This study discusses issues related to the teaching/learning of mathematics and more specifically to the teaching/learning of statistics. Interest in modernizing this area over the past 70 years is evident through numerous legislative adjustments that have taken place to date. However, neither these changes nor the opening to the influence of international educational trends have achieved significant improvements in secondary and university education levels. The results of this study reveal a complex situation in which context, input, process seem to have quite a residual effect on the academic performance, especially in the college level; seemingly other variables could provide a better explanation of the academic performance in this context.
\end{abstract}

Keywords: Mathematics, Statistics, Statistical Literacy, Legislation on education, Attitudes, Academic achievement. 
El lanzamiento por parte de la extinta Unión Soviética del primer satélite artificial de la historia el 4 de octubre de 1957, además de provocar el nerviosismo de los Estados Unidos por su hipotético atraso en la carrera espacial, imprime una mayor preocupación por mejorar el rendimiento de los sistemas educativos occidentales. La consideración de la educación como sector clave para el desarrollo implica la necesidad de asignación de fondos necesarios para lograr este objetivo. Este incremento facilita la investigación y perfeccionamiento de las propuestas didácticas de muy diversa índole que ya venían probándose desde la finalización de la Segunda Guerra Mundial. Cuestiones tales como la administración de los centros educativos, el agrupamiento por nivel de aptitud, la flexibilización de contenidos, la docencia de determinadas materias como lengua o matemáticas o la superdotación son objeto de una atención particular de los investigadores pedagogos y psicólogos y profesionales de la educación. Para ello en unos casos se refuerzan los organismos de estudio y mejora existentes y en otros casos se crean asociaciones especializadas nuevas, como los Institutos de Ciencias de la Educación en España.

En el caso de las matemáticas, ya en 1949 se crea la Comisión Internacional para el estudio y la mejora de la enseñanza de las matemáticas para responder a la necesidad de reforma de esta materia. En 1959, en el Congreso Internacional celebrado en Francia se confirma la necesidad de cambiar de modelo de enseñanza y de contenidos. El cambio toma cuerpo bajo la forma de sugerencias e ideas redactadas por especialistas reunidos en un seminario celebrado en Yugoslavia un año más tarde. Esta propuesta incorpora las aportaciones de los investigadores pedagogos y psicólogos, aunque a veces falte una adecuada comprensión y apropiación de las ideas defendidas por éstos.

\section{Alfabetización matemática en la legislación educativa}

Ley de 1970: Ley 14/1970, de 4 de agosto, General de Educación y Financiamiento de la Reforma Educativa.

Los cambios ocurridos en el mundo llegan a España como parte de esta ley, impulsada por el ministro José Luis Villar Palasí. A este respecto, siguiendo las recomendaciones de los congresos internacionales, destaca la sustitución de la matemática euclídea por la teoría intuitiva de conjuntos. Si en la primera etapa se pretende que los alumnos lleguen al número a través del dominio de las relaciones entre conjuntos, en la segunda se busca una mayor profundización en la expresión matemática formal.

Sin embargo, el hecho de que las autoridades ministeriales no implicaran a los profesionales de la docencia de esta materia en la preparación e implementación de un cambio tan drástico redujo sensiblemente el efecto buscado. La teoría intuitiva de conjuntos, como idealización de la "matemática moderna", se convirtió en un fin en sí mismo en vez de un ser un medio para una mejor comprensión del número, convertido en el modelo a abatir de la "matemática tradicional". No obstante, mientras una parte del profesorado se limita a seguir los libros de texto, pasando por alto los métodos predominantemente activos defendidos en la ley, no faltan quienes prueban modos 
alternativos de enseñar las matemáticas con el objetivo de corregir las deficiencias observadas en las Orientaciones Pedagógicas.

Los resultados de la investigación en torno a la enseñanza de las matemáticas sugieren la necesidad de colmar la creciente brecha que separa las matemáticas del mundo real. En el III Congreso Internacional sobre Educación Matemática celebrado en 1976 en la República Federal de Alemania se confirma la necesidad de una mayor conexión entre estos dos mundos, de utilizar métodos que permitan un aprendizaje activo eminentemente a través de la resolución de problemas recurriendo a las calculadoras para todos los educandos. En España los programas de matemáticas se van concretando más: para los ciclos inicial (1980) y medio (1981) de Enseñanza General Básica estaba previsto incluir temas tales como los conjuntos y correspondencias, la numeración y los conjuntos numéricos, las operaciones, la medida, la geometría y la topología (Sierra Vázquez, 1989). Los cambios previstos para la enseñanza secundaria no llegan a ejecutarse debido a la llegada al poder de nuevos dirigentes políticos y la consiguiente contrarreforma. Aunque se consigue establecer los objetivos mínimos la brecha con respecto al mundo real sigue sin cerrarse. De hecho, el alto nivel de fracaso en matemáticas $( \pm 30 \%)$ así lo sugiere.

En BUP el programa incluye para el primer curso un ambicioso programa que contempla contenidos relativos a combinatoria, números reales, polinomios, funciones polinómicas, ecuaciones, inecuaciones y sistemas, progresiones, estadística y números complejos. Para el segundo curso se imparten sucesiones y límites, funciones reales de variable real, límites y continuidad, funciones trigonométricas, exponencial y logarítmica, derivadas y primitivas, plano vectorial y afín. El tercer curso incluye producto escalar, trigonometría, geometría analítica del plano, números complejos, derivadas, integrales y estadística. En COU se imparten sistemas de ecuaciones lineales, espacios afín y euclídeo tridimensionales, cálculo diferencial e integral y cálculo de probabilidades.

LOGSE (1990): Ley Orgánica 1/1990, de Ordenación General del Sistema Educativo de 3 de octubre de 1990.

Como la de 1970, esta ley llega influida por los acontecimientos científicos del entorno europeo además de los cambios propios de las transformaciones sociopolíticas españolas, especialmente las autonomías. Esta reforma se inspira en diversas propuestas psicopedagógicas desarrolladas en los EEUU como la teoría de los esquemas, el aprendizaje significativo, el cognitivismo, el constructivismo y otras.

Las "cajas rojas" de la LOGSE cumplen el mismo papel que las orientaciones pedagógicas de la reforma anterior: proporcionan orientaciones pedagógicas y metodológicas a los docentes y sirven para difundir los múltiples aspectos de esta reforma. De hecho, incluso la estructura de los libros de vulgarización didáctica está prácticamente calcada sobre las mismas con sus objetivos generales de etapa que se ordenan en conceptos, procedimientos y actitudes, y finalmente contenidos. Ahora bien, la extensión de la obligatoriedad hasta los 16 años provoca la aparición de un tipo de alumnado que hasta entonces era excluido del sistema educativo o derivado a 
la Formación Profesional. Por ello la falta de preparación del profesorado y la imprevisión de las administraciones educativas limitan gravemente los resultados esperados de esta reforma.

Con relación a las matemáticas todos los cursos de la ESO tienen el mismo diseño de bloques de contenidos, a saber números y álgebra, medida y geometría, funciones y gráficas, y estadística y probabilidad. Los mismos cuatro bloques se repiten en Bachillerato con contenidos más avanzados.

Los resultados logrados por la aplicación de esta ley parecen globalmente poco satisfactorios: aunque supuso un revulsivo de primera magnitud para una sociedad en busca de su incorporación al entorno europeo, su impacto educativo deja bastante que desear. En el ámbito de las matemáticas las evaluaciones tanto internas (INCE, 2001) como externas (PISA, 2000, 2003) arrojan resultados más bien desalentadores. Con una muestra de 10.743 alumnos de Educación Primaria el INCE observa, entre otras conclusiones poco halagüeñas, que más de la mitad de los alumnos tiene dificultades con el sistema de numeración decimal, con problemas que implican medidas de tiempo o relacionados con elementos geométricos del plano o representaciones gráficas y cálculo de probabilidades. Resulta fácil adivinar el efecto que esta situación tiene sobre la educación secundaria obligatoria. Por otra parte, la costumbre de dejar para el final la referencia a algunos rudimentos de estadística tanto en primaria como en secundaria, con el conocido peligro que acecha a los últimos temas del curso o del libro de texto, adelanta un olvido casi seguro.

Los informes PISA de 2000 y 2003 no hacen sino confirmar una cierta mediocridad de los resultados: la media española en comprensión lectora, cultura matemática y cultura científica está por debajo de la europea. Peor aún, el nivel de los alumnos españoles de 15 años es tan bajo que en 2000 un $20 \%$ tiene serias dificultades con ejercicios básicos en estas materias. Aparentemente no se trata de una cuestión meramente coyuntural sino más bien estructural. Los resultados de 2003 sitúan a España en el furgón de cola y sin perspectivas de mejora: ya es el $23 \%$ la proporción de estudiantes incapaces de alcanzar el nivel básico en matemáticas. El área de ciencias tampoco se salva de la caída aunque se considere irrelevante estadísticamente. En PISA 2006, la evaluación de la competencia matemática se lleva a cabo en función de cuatro bloques de contenidos (espacio y forma, cambio y relaciones, cantidad, e incertidumbre) que sirven para evaluar ocho habilidades bajo cuatro situaciones graduadas. A juicio de algunos expertos España sigue cosechando malos resultados: retrocede en lectura y se estanca en la mediocridad en ciencias y matemáticas. Sin embargo, en un documento elaborado por su Comisión de Educación (2008), la Real Sociedad Matemática Española (RSME) opina que estos resultados debieran ser analizados con cierta benevolencia puesto que los alumnos no están entrenados para contestar a preguntas redactadas para evaluar sus competencias. Parece sugerirse que la distancia entre lo que los alumnos aprenden en la escuela y la vida real sigue siendo difícil de salvar. 


\section{Nivel de alfabetización matemática - estadística - al acceder a la universidad}

En el número 152 del Boletín Oficial de la Comunidad de Madrid de 27 de junio de 2008 se publica el decreto 67/2008, de 19 de junio, del Consejo de Gobierno, por el que se establece para la Comunidad de Madrid el currículo del Bachillerato. Se formulan como finalidad de la enseñanza de las Matemáticas en el Bachillerato el desarrollo de unas 10 capacidades de las que cabe destacar la $4^{\text {a }}$ que asevera: utilizar las estrategias características de la investigación cientifica y las destrezas propias de las matemáticas (planteamiento de problemas, planificación y ensayo, experimentación, aplicación de la inducción y deducción, formulación y aceptación o rechazo de las conjeturas, comprobación de los resultados obtenidos) para realizar investigaciones y en general explorar situaciones y fenómenos nuevos. A través de este articulado el legislador no oculta su interés por una clara relación entre las matemáticas y la realidad. Define cuatro bloques de contenidos para Matemáticas I siendo el último el de estadística y probabilidad. Además de los detallados contenidos señala doce criterios de evaluación con una expresa mención de la "asignación de "probabilidades a sucesos correspondientes a fenómenos aleatorios simples y compuestos y utilizar técnicas estadísticas elementales para tomar decisiones ante situaciones que se ajusten a una distribución de probabilidad binomial o normal", "la interpretación del grado de correlación existente entre las variables de una distribución estadistica bidimensional sencilla y obtener las rectas de regresión para hacer predicciones estadísticas" y "la realización de investigaciones en las que haya que organizar y codificar informaciones, seleccionar, comparar y valorar estrategias para enfrentarse a situaciones nuevas con eficacia, eligiendo las herramientas matemáticas adecuadas en cada caso".

En cuanto a la enseñanza de las Matemáticas aplicadas a las Ciencias Sociales I y II en el Bachillerato el legislador formula como finalidad el desarrollo de 8 capacidades como la de "aplicación a situaciones diversas los contenidos matemáticos para analizar, interpretar y valorar fenómenos sociales, con objeto de comprender los retos que plantea la sociedad actual". Para ello los contenidos a aprender por parte de los alumnos se explicitan en tres bloques de los cuales el tercero hace referencia a la probabilidad y estadística; incluyen un amplio temario que va desde la estadística descriptiva hasta el contraste de hipótesis para la proporción de una distribución binomial y para la media o diferencias de medias de distribuciones normales con desviación típica conocida. Entre los criterios de evaluación se menciona desde "la interpretación del grado de correlación existente entre las variables de una distribución estadística bidimensional, la obtención de las rectas de regresión para hacer predicciones estadísticas en un contexto de resolución de problemas relacionados con fenómenos económicos y sociales" hasta "el análisis de forma crítica de informes estadísticos presentes en los medios de comunicación y otros ámbitos, y la detección de posibles errores y manipulaciones tanto en la presentación de los datos como de las conclusiones".

Se trata sin duda de un currículo muy ambicioso. Ahora bien, cabe preguntarse si los resultados observados en las evaluaciones de PISA 2000 y 2003 no acaban 
reflejándose al menos parcialmente en la entrada a la universidad. Un indicador del nivel de rendimiento académico del sistema educativo en el área de las matemáticas se encuentra reflejado en el examen de selectividad así como en el número de alumnos que eligen esta carrera universitaria. El hecho de que cada universidad diseñe su propia prueba de selectividad dificulta la comparación pero no impide obtener una imagen general. De entrada, llama la atención que, de manera persistente, las matemáticas se encuentren entre las asignaturas que tienen los peores resultados en todas las universidades tanto por el número de suspensos como por las notas medias. Lo mismo ocurre con aquellas materias que necesitan una cierta base lógicomatemática para su dominio tales como la física o la química. No parece haber diferencias dignas de mención entre los resultados de ciencias en comparación con los de letras en cuanto a la tasa de suspensos.

Las dificultades que se adivinan tras estos resultados tienen como origen un inevitable desajuste que viene de atrás: la complejidad y exigencia de contenidos al pasar de Secundaria a Bachillerato y la amplitud del temario. Todo ello se refleja en un progresivo incremento del rechazo a la opción técnico-científica: del $11 \%$ de alumnos matriculados en esta opción en 2001 se pasa al 8,4\% en 2006; lo cual imposibilita que se consiga aumentar el número de titulados universitarios en ciencias y tecnología como lo reclama la Unión Europea. Por otra parte, no faltan quienes se quejan ante lo que consideran una excesiva laxitud de la prueba de selectividad ya que más del 80\% del alumnado acaba aprobando. De hecho, en el curso 2008-2009, de los 23.052 aspirantes que se presentaron en el distrito único de Madrid 19.941 aprobaron, o sea el $86,50 \%$. En la Universidad Complutense de Madrid se presentaron 8.432 alumnos y aprobaron 7.382, o sea el $87,55 \%$.

Además, parece que la docencia de las matemáticas de Bachillerato está orientada más hacia la superación del examen de selectividad que hacia la preparación para la carrera universitaria. Si se añade a esto la más que probable eliminación acumulada de los últimos temas por falta de tiempo es lógico que ciertos conocimientos se queden sin explicar suficientemente (Estrada, 2001).

\section{Necesidad de alfabetización estadística}

La estadística y la probabilidad como parte integrante de las matemáticas lógicamente sufren el mismo nivel de dominio, o quizás peor, que el cuerpo científico del que son parte. Y si se añade la actitud probablemente negativa transmitida inadvertidamente por el propio docente, debido a la dificultad que él mismo encuentra en la materia o a la escasa importancia que se le otorga en el bagaje académico (Estrada, 2002), se entiende que el nivel de alfabetización estadística del alumnado que ingresa en la universidad sea probablemente deficiente.

\section{Estadística y sociedad}

En la sociedad actual, caracterizada por cambios acelerados, los hechos y sucesos relacionados con la información se crean, distribuyen y manipulan en cualquier contexto sea cultural, político, económico, educativo o social. A este respecto el papel 
de las nuevas tecnologías es esencial: su amplia popularización ha provocado una serie de cambios estructurales prácticamente en todos los niveles de la vida social. Sin embargo, lo relevante no son tanto los hechos y sucesos que difunde como la interpretación que los receptores les asignan.

Con frecuencia a los hechos y sucesos relatados a título de información supuestamente veraz se añaden datos cuya finalidad es reforzar la credibilidad de dicha información. Así los periódicos, las revistas, los telediarios o las páginas webs están repletos de gráficos y cifras que hablan de medias, porcentajes, puntos o décimas de puntos de subida o de caída, etc. Ante este aluvión de datos, los que más saben sobre cuestiones estadísticas tienden a manifestar cierta incredulidad ante la afirmación de que puedan llegar a ser tan transparentes como se presentan mientras quienes no saben sólo pueden apoyarse en la fe ante las afirmaciones de los técnicos. La situación de estos últimos sugiere la necesidad de la formación de cualquier ciudadano para que sea capaz de transformar la información que recibe a través de los medios de comunicación en conocimiento útil para su propio gobierno. Ésta parece haber sido la razón que ha impulsado a los planificadores de la educación a incluir la estadística dentro de los contenidos matemáticos a impartir tanto en Primaria como en Secundaria hasta la universidad. Por tanto no se trata de capacitar al alumno sólo para aplicar propiedades o para resolver algunos ejercicios sino para relacionar la teoría con su realidad social. Además el educando debe ser capaz de recoger, analizar y usar datos para resolver problemas y entender la información del mundo circundante.

\section{La estadística en los estudios universitarios}

A lo largo de las últimas décadas ha resultado cada vez más evidente la necesidad de ampliar la docencia de la estadística y la probabilidad hasta la universidad. De hecho, en el análisis de muchos fenómenos socioeconómicos complejos se recurre con frecuencia a conceptos estadísticos cada vez más sofisticados que exigen un nivel de alfabetización bastante avanzado. Es muy común oír hablar del IPC, del IBEX, del incremento o de la caída del rendimiento académico, de la encuesta de población activa, de la interacción de las actividades humanas con el medio ambiente, etc. Cada uno de estos conceptos implica procedimientos matemático-estadísticos a veces muy complejos y que a su vez exigen un alto nivel de alfabetización para ser capaz de interpretar correctamente los resultados procedentes de la investigación en cada campo especializado. Desde esta perspectiva es lógico que los estudios de una alta proporción de grados incluyan una referencia explícita a la estadística en la denominación de alguna de sus materias. Así, de los 65 grados de la Universidad Complutense de Madrid 44 (68\%) tienen al menos una asignatura obligatoria en cuya denominación aparece el término "estadística" (Tabla 1). La rama de conocimientos de Artes y Humanidades parece ser la única en no contemplar la estadística en la formación de sus estudiantes frente a la de Ciencias Sociales y Jurídicas que alcanza el 92\% de sus grados. 


\begin{tabular}{|c|c|c|c|}
\hline Rama de conocimiento & $\begin{array}{c}\text { Número de } \\
\text { grados }\end{array}$ & $\begin{array}{l}\text { Número de grados con al } \\
\text { menos una materia referida } \\
\text { a la estadística }\end{array}$ & Proporción \\
\hline Artes y Humanidades & 13 & 0 & $0 \%$ \\
\hline Ciencias de la salud & 12 & 9 & $75 \%$ \\
\hline Ciencias & 7 & 6 & $86 \%$ \\
\hline Ingeniería y Arquitectura & 6 & 5 & $83 \%$ \\
\hline Ciencias Sociales y Jurídicas & 24 & 22 & $92 \%$ \\
\hline Dobles títulos & 3 & 2 & $67 \%$ \\
\hline Total & 65 & 44 & \\
\hline
\end{tabular}

Tabla1 La estadística en los grados de la UCM

\section{Actitud ante la estadistica y nivel de ingreso en la universidad}

En general los investigadores coinciden en considerar esencial el papel que juegan las actitudes tanto en el proceso de enseñanza (Estrada et al., 2004, Caballero et al., 2007) como en el aprendizaje de las matemáticas y de la estadística (Pérez-Tyteca et al., 2009). A este respecto, las actitudes se han estudiado desde la perspectiva de sus tres componentes, a saber cognoscitivo, afectivo y relativo a la conducta. Sin embargo, la medición de las actitudes se ha centrado fundamentalmente en el componente cognoscitivo y ha utilizado la encuesta como técnica de recogida de información sobre las opiniones de los sujetos. También hay instrumentos que se centran en el aspecto afectivo para medir entre otras características_individuales la ansiedad (Mondéjar Jiménez et al., 2008), la motivación, las creencias, los valores o las emociones (Caballero et al., 2007). Sin embargo, los distintos instrumentos se diferencian en el número de sus dimensiones así como en los aspectos en los que se centran.

La revisión de los instrumentos más comunes utilizados para este tipo de estudios revela un panorama en el que las características psicométricas no están bien establecidas (Carmona, 2004). Ahora bien, con o sin recurrir a sofisticados instrumentos de medición, los profesores universitarios que imparten materias relacionadas con la estadística saben, sobre todo por experiencia, que la mayoría de sus alumnos llegan literalmente aterrados: muestran un preocupante nivel de ansiedad e inseguridad cuando se enfrentan con ejercicios relativamente sencillos y más aún con exámenes de este tipo de materias. Sin embargo, aparentemente el nivel de implicación de los alumnos muestra una relación negativa con la ansiedad; el componente cognitivo tiene una influencia menos clara (Mondéjar et al., 2008).

Con relación al entorno universitario muchos estudios están interesados en lo que les ocurre a los alumnos futuros profesores de matemáticas mientras se preparan en las aulas universitarias. Algunos resultados parecen confirmar la creencia adquirida a lo largo de la experiencia escolar de que las matemáticas son una herramienta útil y necesaria para cualquier ciudadano de la sociedad del conocimiento y además para el dominio de otras disciplinas relacionadas con ella. Estos alumnos parecen ser capaces de percibir la relación que existe entre las matemáticas y la realidad. Sin embargo, otros estudios obtienen resultados contrarios que muestran las matemáticas como una materia ardua, aburrida y alejada de la realidad o que los futuros docentes de Primaria no tienen un dominio suficiente de los conceptos elementales relacionados con la estadística (Estrada y Batanero, 2008). 
¿Qué pasa con los alumnos, generalmente de Letras, que hacen todo lo posible por evitar las matemáticas o cualquier contenido que haga referencia al número o al razonamiento lógico-matemático en Bachillerato? Además de su alto nivel de ansiedad, estos alumnos se caracterizan, a juicio de Peñaloza Figueroa \& Vargas Pérez (2006), por la no utilización de códigos elaborados, la pobreza de las competencias relativas al uso de términos sintácticos, las bajas capacidades y destrezas operativas y de cálculo, sus bajos niveles de motivación intrínseca, la ausencia de inter-relación entre lo que estudian y el mercado laboral y sus metas de corto plazo (McKenzie \& Schweitzer, 2001).

Puesto que las asignaturas de estadística y similares están incluidas en los planes de estudios como troncales u obligatorias, el alumno se ve abocado a hacer frente a esta situación lo mejor que puede. Dado que aprobar es más importante que aprender el alumno tiende a recurrir a la pura y simple memorización de los apuntes de clase así como a la resolución mecánica de ejercicios que en la mayoría de los casos considera ajenos a su mundo. Generalmente las advertencias de los profesores respecto de las estrategias de estudio de esta materia suelen tener muy poco éxito. Incluso las clases magistrales acompañadas de aplicaciones prácticas se convierten en un tedioso ejercicio de resistencia tanto para los profesores como para los alumnos; los proyectos individuales o grupales supervisados por el profesor apenas pasan de ser tareas que se llevan a cabo como un mero trámite formal conducente al ansiado aprobado. La experimentación como vehículo para el aprendizaje recibe una escasísima atención puesto que no garantiza el aprobado; lo mismo le ocurre a las clases orientadas a la formulación de conjeturas apropiadas para ser contrastadas empíricamente o a la identificación de problemas susceptibles de ser investigados experimentalmente (Lizzio et al., 2002). En cuanto a la información presente en la vida diaria del alumnado bajo la forma de textos, artículos especializados o no, la principal dificultad radica en el bajo nivel de comprensión lectora así como en el hecho de que los alumnos no perciban su relación con el aprobado.

\section{Método}

Los numerosos estudios llevados a cabo a lo largo de las últimas décadas y centrados en variables que afectan al rendimiento académico en la enseñanza universitaria y al abandono (Bruinsma, 2003; McInnis et al., 2000) han combinado diversos enfoques: psicológico, organizacional, económico, social, interactivo y de eficacia escolar. Las principales variables estudiadas están relacionadas con las características del contexto, de entrada de profesores y alumnos y de los procesos. Entre las características del estudiante más estudiadas cabe destacar los conocimientos previos, el sexo, la edad, la motivación, las actividades de estudio y el estilo de aprendizaje (Hativa \& Birenbaum, 2000). En cuanto al profesor los investigadores se han centrado, entre otros aspectos, en los valores y creencias, el conocimiento, el pensamiento, las destrezas comunicativas, las habilidades de ejecución y la personalidad. Las variables de proceso se han operacionalizado tanto para el profesor como para el alumno como el comportamiento de ambos en la interacción educativa. Las variables de contexto se han definido como aquellas que son externas al aula y que sin embargo afectan a los docentes y a los estudiantes con relación a los procesos de aula y a los resultados. 
A pesar de la gran cantidad de estudios sobre los factores que afectan al rendimiento académico y al abandono, aún queda profundizar en algunos aspectos (Pike \& Saupe, 2002). Los modelos de investigación aplicados hasta ahora han concedido una notable atención a los aspectos cuantitativos (número de horas dedicados al estudio, por ejemplo) en detrimento de los cualitativos (estructura y organización del curso, por ejemplo). Además, tienden a descuidar la relación que existe entre la motivación del estudiante, el procesamiento profundo de la información y la calidad del ambiente de aprendizaje, por una parte, y por otra la relación entre estas dos variables y el rendimiento (Eppler \& Harju, 1997). Finalmente, los diseños utilizados suelen ser de tipo transversal o de laboratorio. Ya que los estudios universitarios se adecuan a un proceso longitudinal, un diseño de investigación de características similares parece más aconsejable: proporcionaría estimaciones más precisas y probablemente más fiables.

El presente estudio toma en consideración los tres aspectos antes mencionados e integra variables relacionadas con tres líneas de investigación para determinar el contexto, la entrada y los factores procesuales de aula que pueden afectar al rendimiento. Las tres líneas de investigación en las que se inspira son: (a) la calidad de la interacción educativa y su relación con la decisión del estudiante de abandonar o de proseguir; (b) la eficacia escolar y la productividad educativa; (c) el carácter mediador de la calidad del procesamiento de la información entre la motivación en tanto que expectativas, valores y afecto, y el rendimiento.

Este estudio tiene por finalidad evaluar la eficacia diferencial de tres métodos de enseñanza/aprendizaje de contenidos relativos a la estadística especialmente en el contexto de la formación de los pedagogos, habida cuenta las características del contexto y de entrada y a lo largo de todo un curso académico. Este estudio se lleva a cabo con los datos procedentes de los alumnos de $2^{\circ}$ de Pedagogía de la facultad de Educación de la Universidad Complutense de Madrid. Las variables implicadas en el diseño son:

\begin{tabular}{|l|l|c|c|}
\hline \multicolumn{1}{|c|}{ Contexto } & \multicolumn{1}{c|}{ Entrada } & \multicolumn{1}{c|}{ Proceso } & \multicolumn{1}{c|}{ Producto } \\
\hline $\begin{array}{l}\text { Cohorte, docente, } \\
\text { horario, contenidos }\end{array}$ & $\begin{array}{l}\text { Conocimientos previos y } \\
\text { Actitudes (Actitud, Dificultad e } \\
\text { Interés) }\end{array}$ & $\begin{array}{l}\text { Métodos } \\
\text { didácticos }\end{array}$ & Rendimiento \\
\hline
\end{tabular}


El diseño se ejecuta según la siguiente pauta (Fig. 1):

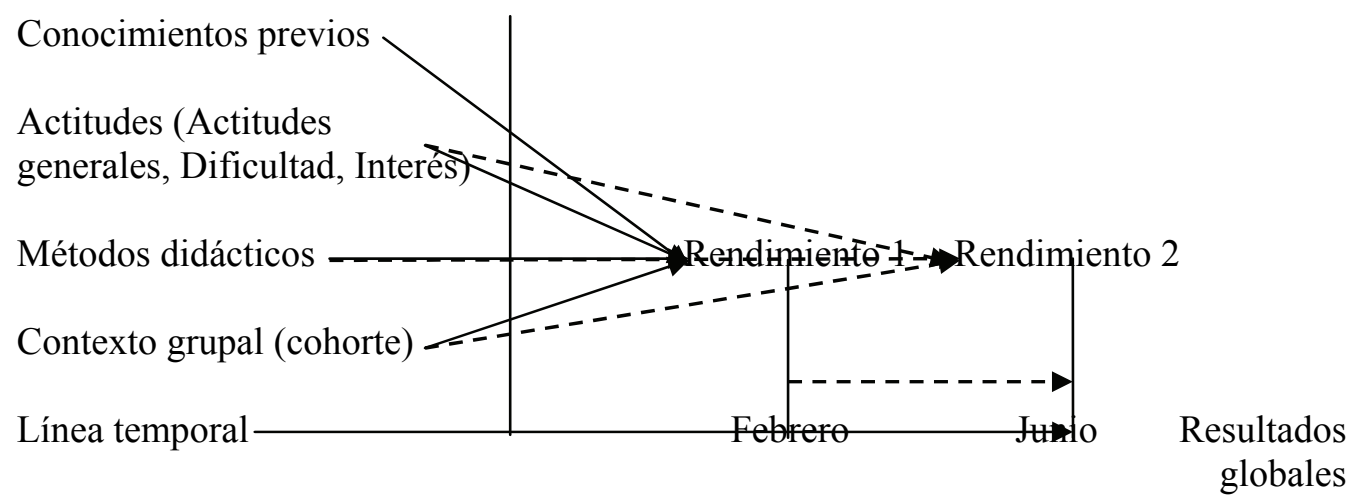

\section{Fase $1 \quad$ Fase 2}

Fase 3

Fase 4

Fig. 1. Variables nucleares y secuencia del diseño.

La fase 1 coincide con el principio del curso: se recogen datos sobre los conocimientos previos y las actitudes para cada cohorte. Para ello, los alumnos son informados de que tras un mes y medio de clase han de contestar un cuestionario de actitud hacia la estadística y además realizar una prueba de conocimientos previos; los alumnos saben que la información procedente de estas pruebas les debe servir para que cada uno conozca su situación inicial; se trata por lo tanto de una evaluación puramente diagnóstica, sin consecuencia alguna para aprobar o suspender la materia. Con relación a la primera prueba se selecciona y aplica la Escala de Actitudes Hacia la Estadística (Muñoz San Roque, 2002) debido al alto nivel de fiabilidad reportado por su autora en cada una de sus 5 dimensiones, a saber: competencia percibida por el propio alumno sobre su habilidad hacia la estadística, agrado mostrado hacia la asignatura de estadística, utilidad de la estadística para el futuro profesional, dificultad de la estadística como asignatura que está cursando y dificultad de la estadística como campo de estudio. Los datos aportados por esta escala se completan con una percepción global de cada alumno respecto del nivel esperado de dificultad y de interés de esta materia en una escala de 0 a 10 . La segunda prueba se diseña a partir del contenido de diversos manuales de Filosofía y de Matemáticas de $1^{\circ}$ y $2^{\circ}$ de Bachillerato. A este respecto los alumnos saben que se hará la comprobación de su nivel de dominio de la lógica básica, probabilidad, muestreo, inferencia y correlación. A partir del curso 2008-2009, debido a las dificultades manifestadas por los alumnos, esta prueba es sustituida por otra más sencilla diseñada a partir de contenidos recogidos en los libros de Matemáticas de $3^{\circ}$ y $4^{\circ}$ de ESO y centrada en dos dimensiones, a saber las bases de estadística descriptiva y probabilidad.

En este momento y para el estudio de esta materia a lo largo del curso los alumnos eligen entre tres opciones no excluyentes: (a) Asistencia a las tradicionales clases teóricas que se complementan con algún ejercicio práctico, (b) Trabajos realizados y entregados mediante la plataforma WebCt (Campus Virtual) y (c) Ejercicios de solución de problemas; la opción (b) se ofrece a un máximo de 20 alumnos. La opción (c) se lleva a cabo con la colaboración de monitores voluntarios de $4^{\circ}$ o $5^{\circ}$ curso; estos 
monitores son entrenados para que sean capaces de ayudar a los alumnos en la realización de unos ejercicios prácticos semanales bajo la supervisión del profesor. En suma, cada alumno puede elegir una de las tres opciones o compaginarlas sabiendo que al final tendrá que someterse a los tradicionales exámenes obligatorios para aprobar. Ahora bien, si bien este modo de actuar permite captar las preferencias de los alumnos, a la vez impide la formación de subgrupos equilibrados.

Todos estos datos son utilizados para comprobar si hay diferencias iniciales relevantes en las características de entrada entre los alumnos a la hora de elegir entre las tres opciones. A esta información inicial se añade el número de convocatorias agotadas. Además, se solicita que cada alumno indique si se identifica con el área de Ciencias o Letras o la modalidad de Bachillerato que ha cursado (Artes, Ciencias de la Naturaleza y de la Salud, Humanidades y Ciencias Sociales, y Tecnología).

La fase 2 coincide con la convocatoria oficial de exámenes de febrero. Los datos relativos al rendimiento académico se recogen a través de los tradicionales exámenes de este periodo. La validez instructiva de los mismos explica que éstos fueran elegidos en lugar de recurrir a pruebas estandarizadas. Cada examen consta de dos partes: la primera evalúa el dominio de la fundamentación teórica con una prueba de 40-60 ítems de opción múltiple y la segunda la capacidad del discente de relacionar la teoría y la práctica, a través de ejercicios diseñados tomando el contexto socioeducativo como única referencia. Aunque las pruebas teóricas no fueron sometidas a un estricto proceso de estandarización tienen un nivel de fiabilidad cuyo alfa de Cronbach alcanza los siguientes valores:

\begin{tabular}{|l|c|c|c|c|c|}
\hline Curso & $\mathbf{2 0 0 5 - 2 0 0 6}$ & $\mathbf{2 0 0 6 - 2 0 0 7}$ & $\mathbf{2 0 0 7 - 2 0 0 8}$ & $\mathbf{2 0 0 8 - 2 0 0 9}$ & $\mathbf{2 0 0 9 - 2 0 1 0}$ \\
\hline Alfa & 0,677 & 0,620 & 0,547 & 0,731 & 0,654 \\
\hline N & 77 & 61 & 63 & 49 & 79 \\
\hline
\end{tabular}

Tabla 2. Nivel de fiabilidad de las pruebas teóricas

La relativamente baja fiabilidad de este instrumento podría deberse a dos razones: (a) el examen está diseñado para evaluar la comprensión de los contenidos explicados en clase así como la aplicación de los principios teóricos más importantes presentados en el aula; la memorización tiene un peso que no llega al 10\%. Además, la amplitud de contenidos expuestos permite el diseño de ítems suficientemente variados y no necesariamente relacionados entre sí. (b) Por otra parte, obviamente el objetivo del examen no es tanto diferenciar entre los que más dominan la materia y los que no como averiguar el nivel de dominio de cada uno. La escasa variación sólo evidencia el alto nivel de semejanza que tienen estos alumnos ante esta materia.

En esta fase se comprueba la relación que existe entre las características de entrada a los métodos y el rendimiento.

La fase 3 coincide con la convocatoria oficial de exámenes de junio. Se aplica el mismo procedimiento que en febrero. Además se pide a los alumnos que evalúen el nivel experimentado de dificultad y de interés en la escala de 0 a 10, como al principio del curso.

En la fase 4 se evalúa la evolución de una parte de las características de entrada, a saber el nivel de dificultad y de interés y del producto, el rendimiento. 


\section{Hipótesis sustantiva}

Las características de contexto, entrada y proceso relacionadas con el nivel de rendimiento de los alumnos en la materia.

\section{Subhipótesis:}

Con relación esta materia:

1. El nivel de conocimientos previos explica al menos parcialmente el nivel de rendimiento de los alumnos.

2. La percepción que los alumnos tienen de esta materia explica al menos parcialmente su nivel de rendimiento.

3. La modalidad de Bachillerato (Artes, Ciencias de la Naturaleza y de la Salud, Humanidades y Ciencias Sociales, y Tecnología) condiciona el nivel de rendimiento en esta materia.

4. El número de convocatorias agotadas está relacionado con el nivel de rendimiento discente.

5. Los métodos de enseñanza/aprendizaje elegidos por los alumnos explican al menos parcialmente el nivel de rendimiento.

6. Los alumnos rinden más en la parte práctica que en la parte teórica de la materia.

7. La percepción del nivel de dificultad e interés que tienen los alumnos varía sensiblemente a lo largo del curso.

8. La cohorte a la que pertenecen los alumnos es un factor explicativo relevante de las diferencias observadas en el rendimiento parcial y final.

\section{Muestra}

La muestra la componen todos los alumnos matriculados en el grupo de clase desde el curso 2005-2006 hasta 2009-2010. Son 528 y se distribuyen como sigue de acuerdo con el cómputo de la matrícula oficial:

\begin{tabular}{|l|c|r|r|r|c|}
\hline Curso & 2005-2006 & 2006-2007 & 2007-2008 & 2008-2009 & 2009-2010 \\
\hline Total inicial & 109 & 103 & 97 & 93 & 126 \\
\hline $\begin{array}{l}\text { Alumnos presentados en } \\
\text { la convocatoria de } \\
\text { Febrero }\end{array}$ & 77 & 61 & 63 & 49 & 79 \\
\hline$\%$ de presentados & $71 \%$ & $59 \%$ & $65 \%$ & $53 \%$ & $63 \%$ \\
\hline $\begin{array}{l}\text { Alumnos presentados en } \\
\text { la convocatoria de Junio }\end{array}$ & 59 & 37 & 43 & 34 & 50 \\
\hline$\%$ de presentados & $54 \%$ & $36 \%$ & $44 \%$ & $37 \%$ & $40 \%$ \\
\hline $\begin{array}{l}\text { Diferencia porcentual } \\
\text { entre Junio y Febrero }\end{array}$ & $-17 \%$ & $-23 \%$ & $-21 \%$ & $-16 \%$ & $-23 \%$ \\
\hline
\end{tabular}

Tabla 3. Distribución del número de alumnos por curso.

La casi totalidad del alumnado (98\%-99\%) afirma que eligió cursar esta carrera. Por término medio aproximadamente el $60 \%$ de los alumnos matriculados se presenta 
a los exámenes parciales de febrero de esta materia. Los que toman parte en la convocatoria de junio apenas superan el 40\%. Esto significa que en realidad en torno al $60 \%$ de los alumnos o nunca se presenta a examen (40\%) o abandona la materia $(20 \%)$ tras el primer examen.

\section{Resultados}

El análisis de los datos recurre inicialmente a los estadísticos descriptivos mientras el contraste de hipótesis utiliza las pruebas t de Student, F, H de Kruskal-Wallis. Se considera estadísticamente significativo cualquier valor cuya probabilidad asociada es igual o inferior a 0,05 . Para las pruebas relativas al efecto diferencial de los métodos se añade el valor correspondiente a la magnitud del efecto: $\eta^{2}$ para $F$ y d (Cohen) para t.

\section{Fase I: Situación inicial}

\section{Actitud hacia la estadística}

Contrariamente a la información proporcionada por su autora la prueba de 34 ítems que se utilizó arroja un nivel de fiabilidad global muy alejada de 0,90; las cinco aplicaciones consecutivas arrojan un índice que oscila entre 0,106 y 0,331 para muestras cuyo tamaño varía entre 54 y 86 alumnos por curso.

\begin{tabular}{|l|c|c|c|c|c|}
\hline Curso & 2005-2006 & 2006-2007 & 2007-2008 & 2008-2009 & 2009-2010 \\
\hline Alfa de Cronbach & 0,244 & 0,106 & 0,288 & 0,331 & 0,109 \\
\hline \% de la varianza FI & 40,725 & 37,682 & 35,968 & 40,389 & 36,140 \\
\hline $\mathrm{N}$ & 78 & 72 & 70 & 54 & 86 \\
\hline
\end{tabular}

Tabla 4. Nivel de fiabilidad de la prueba de actitud ante la estadística

Esta prueba sufre de la no resuelta debilidad de las características psicométricas de los instrumentos de este contexto, a la que Carmona (2004) hace referencia. No obstante, en este estudio no se busca identificar diferencias en actitud ante la estadística sino establecer el nivel actitudinal individual en un contexto en el que la similitud entre los sujetos es la característica más sobresaliente. Por ello, la información que se obtiene se utiliza para averiguar si ese nivel está relacionado con la elección entre los tres métodos para enfrentarse al aprendizaje de esta materia. Para ello se utilizan las puntuaciones del primer factor obtenido al aplicar un análisis factorial de componentes principales con rotación Varimax: se trata de un factor general bipolar que proporciona una información global sobre la posición de cada alumno en el continuo de actitud negativa-positiva hacia la estadística. Globalmente no son significativas las diferencias actitudinales que hay entre los alumnos a la hora de elegir un método u otro (Tabla 5). Lo mismo ocurre respecto del nivel de dificultad y de interés que creen va a tener la materia. El factor I tiende a correlacionar bajo pero positiva y significativamente con el nivel de dificultad y negativamente con el nivel de interés (Tabla 6). 


\begin{tabular}{|c|l|c|c|c|c|c|}
\hline \multicolumn{2}{|c|}{} & $\mathbf{2 0 0 5 - 2 0 0 6}$ & $\mathbf{2 0 0 6 - 2 0 0 7}$ & $\mathbf{2 0 0 7 - 2 0 0 8}$ & $\mathbf{2 0 0 8 - 2 0 0 9}$ & $\mathbf{2 0 0 9 - 2 0 1 0}$ \\
\hline \multirow{4}{*}{$\begin{array}{c}\text { Factor I - } \\
\text { Actitud }\end{array}$} & $\begin{array}{l}\text { Media } \\
\text { general }\end{array}$ & 0,022 & $-0,022$ & $-0,001$ & $-0,000$ & 0,046 \\
\cline { 2 - 7 } & $\mathrm{F}$ & 7,722 & 1,129 & 1,639 & 2,112 & 1,058 \\
\cline { 2 - 7 } & $p$ &, 000 &, 311 &, 180 &, 110 &, 353 \\
\hline \multirow{3}{*}{$\begin{array}{c}\text { Nivel inicial de } \\
\text { dificultad }\end{array}$} & $\begin{array}{l}\text { Media } \\
\text { general }\end{array}$ & 7,93 & 8,17 & 8,04 & 7,46 & 6,42 \\
\cline { 2 - 7 } & $\mathrm{F}$ & 1,466 & 2,441 & 0,926 & 1,623 & 1,500 \\
\cline { 2 - 7 } & $p$ &, 229 &, 088 &, 420 &, 119 &, 280 \\
\hline \multirow{3}{*}{$\begin{array}{c}\text { Nivel inicial de } \\
\text { interés }\end{array}$} & $\begin{array}{l}\text { Media } \\
\text { general }\end{array}$ & 5,63 & 5,52 & 5,59 & 6,37 & 5,84 \\
\cline { 2 - 7 } & $\mathrm{F}$ & 2,573 & 1,057 & 0,996 & 3,003 & 3,361 \\
\cline { 2 - 7 } & $p$ &, 059 &, 372 &, 417 & $\mathbf{0 3 6}$ & $\mathbf{0 2 3}$ \\
\cline { 2 - 7 } & $\mathrm{N}$ & 92 & 82 & 68 & 81 & 85 \\
\hline
\end{tabular}

Tabla 5. Probabilidad asociada a las diferencias relativas a la elección entre los métodos de aprendizaje.

\begin{tabular}{|l|l|c|c|c|c|c|}
\hline \multicolumn{2}{|c|}{ Curso } & $\mathbf{2 0 0 5 - 2 0 0 6}$ & $\mathbf{2 0 0 6 - 2 0 0 7}$ & $\mathbf{2 0 0 7 - 2 0 0 8}$ & $\mathbf{2 0 0 8 - 2 0 0 9}$ & $\mathbf{2 0 0 9 - 2 0 1 0}$ \\
\hline Nivel inicial de & $r_{s}$ &, 564 &, 384 &, 384 &, 086 &, 333 \\
\cline { 2 - 7 } Dificultad & $p$ & $\mathbf{, 0 0 0}$ & $\mathbf{, 0 0 5}$ & $\mathbf{, 0 1 3}$ &, 555 &, $\mathbf{0 1 1}$ \\
\hline \multirow{2}{*}{$\begin{array}{l}\text { Nivel inicial de } \\
\text { Interés }\end{array}$} & $r_{s}$ &,- 316 &,- 377 &,- 126 &,- 506 &,- 189 \\
\cline { 2 - 7 } & $p$ &, 007 &, 005 &, 433 &, 000 &, 155 \\
\hline
\end{tabular}

Tabla 6. Correlación entre Factor I, y Dificultad e Interés.

\section{Conocimientos previos}

La evaluación diagnóstica de los conocimientos previos se lleva a cabo mediante una prueba de 40 ítems de opción múltiple. Los ítems se reparten del siguiente modo:

\begin{tabular}{|c|c|c|c|c|c|c|c|}
\hline Curso & N & $\begin{array}{c}\text { Lógica } \\
\text { 15 ítems } \\
\text { Media }\end{array}$ & $\begin{array}{c}\text { Probabilidad } \\
\text { 8 ítems Media }\end{array}$ & $\begin{array}{c}\text { Muestreo } \\
\mathbf{7} \text { ítems } \\
\text { Media }\end{array}$ & $\begin{array}{c}\text { Inferencia } \\
\text { 5 ítems } \\
\text { Media }\end{array}$ & $\begin{array}{c}\text { Correlación } \\
\text { 5 ítems } \\
\text { Media }\end{array}$ & $\begin{array}{c}\text { Media } \\
\text { global } \\
\text { sobre 10 }\end{array}$ \\
\hline $2005-06$ & 92 & 7,50 & 4,29 & 1,10 & 2,13 & 2,07 & 4,27 \\
\hline $2006-07$ & 82 & 7,11 & 3,24 & 0,77 & 1,57 & 1,63 & 3,50 \\
\hline $2007-08$ & 68 & 7,06 & 3,81 & 0,87 & 2,11 & 1,94 & 3,92 \\
\hline
\end{tabular}

Tabla 7. Nivel medio de conocimientos previos.

Los alumnos contestan a preguntas similares a las siguientes:

(a) Sólo uno de los siguientes razonamientos condicionales tiene una forma válida.

1) Si llueve, las calles se mojan. No llueve, luego las calles no se mojan.

2) Si llueve, las calles se mojan. Las calles se mojan, luego llueve.

3) Si llueve, las calles se mojan. Llueve, luego las calles se mojan.*

4) Si llueve, las calles se mojan. Llueve, luego las calles no se mojan. 
(b) Si se repitiera el mismo evento un número infinito de veces, ¿en qué caso se obtendría una distribución binomial?

1) Lanzar dados.

2) Lanzar monedas.*

3) Extraer cartas de una baraja.

4) Extraer bolas de un bombo.

En el caso de los cursos 2008-2009 y 2009-2010 la prueba consta de 65 ítems del tipo verdadero/falso que se reparten del siguiente modo:

\begin{tabular}{|c|c|c|c|c|}
\hline Curso & $\mathbf{N}$ & $\begin{array}{c}\text { Bases de estadística } \\
\text { 35 ítems } \\
\text { Media }\end{array}$ & $\begin{array}{c}\text { Bases de probabilidad } \\
\text { 30 ítems } \\
\text { Media }\end{array}$ & $\begin{array}{c}\text { Media global } \\
\text { sobre 10 }\end{array}$ \\
\hline $2008-09$ & 81 & 27,28 & 20,52 & 7,317 \\
\hline $2009-10$ & 85 & 27,24 & 22,71 & 7,683 \\
\hline
\end{tabular}

Tabla 8. Nivel medio de conocimientos previos.

Contestan a preguntas similares a las siguientes:

(a) Si el director de un centro educativo quiere saber si gusta su modo de dirigir y pregunta a una parte de los profesores y alumnos, entonces debe considerar a aquéllos quienes pregunta como

1) Su muestra.*

2) Su población

(b) ¿Cuál de las siguientes afirmaciones sobre la frecuencia relativa de los experimentos aleatorios es correcta?

1) El suceso seguro tiene una frecuencia relativa o probabilidad igual a 0 .

2) La frecuencia relativa o probabilidad de un suceso tiene un valor entre $0 \mathrm{y}$ $1 . *$

Cuando el contenido de la prueba hace referencia a lo que teóricamente los alumnos deberían haber estudiado en Bachillerato resulta que el nivel de sus conocimientos previos está por debajo de la media de la escala, 5. En cambio, cuando el contenido incluye cuestiones estadísticas relativas a $3^{\circ}$ y $4^{\circ}$ de ESO, entonces su nivel está por encima de la media de la escala. Esta situación sugiere la existencia de una notable distancia entre el currículum oficial de Bachillerato y los contenidos que finalmente estudian al menos con relación a los fundamentos de probabilidad, muestreo, inferencia y correlación. El nivel de conocimientos de los contenidos de ESO parece responder sobre todo al estadio evolutivo del alumnado. El nivel medio de maduración lógico-matemática y de alfabetización estadística de estos alumnos se encuentra por debajo de lo esperado al inicio del segundo curso de su carrera universitaria. Por otra parte a este respecto los alumnos de las cuatro modalidades de Bachillerato tienen un nivel prácticamente inicial similar (Tabla 9). 


\begin{tabular}{|l|l|c|c|c|c|c|}
\hline \multicolumn{2}{|c|}{ Curso } & $\mathbf{2 0 0 5 - 2 0 0 6}$ & $\mathbf{2 0 0 6 - 2 0 0 7}$ & $\mathbf{2 0 0 7 - 2 0 0 8}$ & $\mathbf{2 0 0 8 - 2 0 0 9}$ & $\mathbf{2 0 0 9 - 2 0 1 0}$ \\
\hline \multirow{4}{*}{$\begin{array}{l}\text { Conocimientos } \\
\text { previos }\end{array}$} & $\begin{array}{l}\text { Media } \\
\text { general }\end{array}$ & 4,267 & 3,503 & 3,923 & 7,317 & 7,683 \\
\cline { 2 - 7 } & $\mathrm{F}$ & 0,563 & 1,666 & 3,488 & 0,593 & 0,400 \\
\cline { 2 - 7 } & $p$ & 0,641 & 0,181 & $\mathbf{0 , 0 1 2}$ & 0,622 & 0,753 \\
\cline { 2 - 7 } & $\mathrm{N}$ & 92 & 82 & 68 & 81 & 85 \\
\hline
\end{tabular}

Tabla 9. Probabilidad asociada a las diferencias relativas a la elección entre los métodos de aprendizaje.

En resumen, al inicio del curso los alumnos destacan por su notable nivel de semejanza en las características de entrada.

\section{Fase II: Resultados de la evaluación de febrero}

La evaluación de la parte teórica se lleva a cabo mediante una prueba de 40 ítems de elección múltiple que revisa la fundamentación teórico-práctica esencial de la investigación educativa. A este respecto los alumnos tienen que responder a preguntas similares a las siguientes:

(a) Una variable dependiente se define como una variable

1) No controlada que varía simultáneamente con los cambios en los niveles de la variable manipulada

2) Conocida que influye sobre la variable manipulada y cuyo efecto anula el investigador

3) Receptora del efecto de la variable manipulada por el investigador*

4) Utilizada para emparejar los grupos

(b) Al relacionar dos variables continuas de una muestra se obtiene como resultado 0,76 ; se averigua que este valor tiene una probabilidad de ocurrir por azar de 0,06. Como el investigador fijó como nivel de error 0,06, debe

1) Aceptar $H_{0}$ pudiendo cometer un error del Tipo II

2) Rechazar $H_{0}$ pudiendo cometer un error del Tipo II

3) Rechazar $H_{0}$ pudiendo cometer un error del Tipo I*

4) Evitar rechazar o aceptar $H_{0}$ por carecer de valor crítico

El dominio de la parte práctica se evalúa mediante un único problema enmarcado en el entorno educativo. Sobre este problema se plantean varias preguntas cuyo objetivo es comprobar hasta qué punto el alumnado es capaz de traducir los conceptos teóricos en operaciones concretas tales como averiguar la probabilidad de obtener una determinada media por azar o si un determinado índice de correlación es aleatorio o no e interpretarlo. La comparación de las medias obtenidas por los alumnos de las distintas opciones de aprendizaje muestra que el rendimiento es bastante similar sobre todo en la parte teórica (Tabla 10). Sin embargo, los índices de correlación entre los dos aspectos apenas superan 0,500 a lo largo de los cursos pero indican una relación altamente significativa que es por término medio moderada. 


\begin{tabular}{|l|l|c|c|c|c|c|}
\hline \multicolumn{2}{|l}{ Curso } & $\mathbf{2 0 0 5 - 2 0 0 6}$ & $\mathbf{2 0 0 6 - 2 0 0 7}$ & $\mathbf{2 0 0 7 - 2 0 0 8}$ & $\mathbf{2 0 0 8 - 2 0 0 9}$ & $\mathbf{2 0 0 9 - 2 0 1 0}$ \\
\hline \multirow{4}{*}{ Teoría 1 } & Media general & 5,011 & 4,381 & 4,429 & 4,786 & 3,823 \\
\cline { 2 - 7 } & $F$ & 1,166 & 1,538 &, 426 &, 340 &, 752 \\
\cline { 2 - 7 } & $p$ &, 329 &, 214 &, 789 &, 797 &, 475 \\
\cline { 2 - 7 } & $\eta^{2}$ & 0,046 &, 075 &, 029 &, 022 &, 020 \\
\hline \multirow{5}{*}{ Práctica 1} & Media general & 3,857 & 4,416 & 4,687 & 5,054 & 4,553 \\
\cline { 2 - 7 } & $F$ & 2,491 & 6,287 & 3,593 & 2,091 & 4,034 \\
\cline { 2 - 7 } & $p$ &, 067 &, 001 &, 011 &, 115 &, 022 \\
\cline { 2 - 7 } & $\eta^{2}$ &, 093 &, 249 &, 199 &, 122 &, 097 \\
\hline & $r_{\text {TeoriaxPráctica }}$ &, 772 &, 429 &, 607 &, 469 &, 374 \\
\cline { 2 - 7 } & $P$ & $\mathbf{, 0 0 0}$ & $\mathbf{, 0 0 1}$ & $\mathbf{, 0 0 0}$ & $\mathbf{, 0 0 1}$ &, $\mathbf{0 0 1}$ \\
\cline { 2 - 7 } & $\mathrm{N}$ & 77 & 61 & 63 & 49 & 79 \\
\hline
\end{tabular}

Tabla 10. Probabilidad asociada a las diferencias de rendimiento en febrero con relación a los métodos de aprendizaje.

Por otra parte, independientemente de que los conocimientos previos se midan con relación a la ESO o a Bachillerato, su correlación con el rendimiento teórico y práctico tiende a ser baja o muy baja y no significativa (Tabla 11).

\begin{tabular}{|c|c|c|c|c|c|c|c|c|c|c|}
\hline \multirow[t]{2}{*}{ Curso } & \multicolumn{2}{|c|}{ 2005-2006 } & \multicolumn{2}{|c|}{ 2006-2007 } & \multicolumn{2}{|c|}{ 2007-2008 } & \multicolumn{2}{|c|}{ 2008-2009 } & \multicolumn{2}{|c|}{ 2009-2010 } \\
\hline & $r_{x y}$ & $p$ & $r_{x y}$ & $p$ & $r_{x y}$ & $p$ & $r_{x y}$ & $p$ & $r_{x y}$ & $p$ \\
\hline Teoría 1 & .380 & 001 & 397 &, 002 &,- 006 & 968 & .289 & 044 &,- 077 & .557 \\
\hline Práctica 1 & ,034 &, 778 &,- 107 &, 430 &,- 048 &, 732 &, 340 & ,017 &,- 169 &, 196 \\
\hline $\mathrm{N}$ & \multicolumn{2}{|c|}{77} & \multicolumn{2}{|c|}{61} & \multicolumn{2}{|c|}{63} & \multicolumn{2}{|c|}{49} & \\
\hline
\end{tabular}

Tabla 11. Probabilidad asociada a la relación entre el nivel de conocimientos previos y el rendimiento en esta materia en febrero.

La dificultad e interés manifestados por el alumnado no aparecen como factores relevantes relacionados con el nivel de rendimiento global. Al igual que en el caso anterior el rendimiento se relaciona de manera débil o no significativa con el nivel de dificultad e interés (Tabla 12).

\begin{tabular}{|c|c|c|c|c|c|c|c|c|c|c|}
\hline \multirow[t]{2}{*}{ Curso } & \multicolumn{2}{|c|}{$2005-2006$} & \multicolumn{2}{|c|}{ 2006-2007 } & \multicolumn{2}{|c|}{$2007-2008$} & \multicolumn{2}{|c|}{ 2008-2009 } & \multicolumn{2}{|c|}{ 2009-2010 } \\
\hline & $r_{s}$ & $p$ & $r_{s}$ & $p$ & $r_{s}$ & $p$ & $r_{s}$ & $p$ & $r_{s}$ & $p$ \\
\hline Dificultad 1 & ,023 &, 845 &, 075 &, 581 &,- 038 &, 793 &,- 232 &, 108 &,- 315 & ,014 \\
\hline Interés 1 &,- 269 &, 021 &, 113 &, 409 &, 169 & 241 &, 310 &, 030 &,- 003 & ,984 \\
\hline $\mathrm{N}$ & \multicolumn{2}{|c|}{73} & \multicolumn{2}{|c|}{56} & \multicolumn{2}{|c|}{50} & \multicolumn{2}{|c|}{49} & \multicolumn{2}{|c|}{60} \\
\hline
\end{tabular}

Tabla 12. Relación entre el rendimiento global de febrero y el nivel de dificultad e interés.

El tipo de estudios cursados en Bachillerato clasificado como de Ciencias o Letras o en las cuatro categorías al uso no implica diferencias claras por sí sólo con relación al nivel de rendimiento global (teoría y práctica). Incluso las diferencias observadas en los últimos dos cursos pueden ser meramente coyunturales porque en ellas parecen influir en gran medida la composición del alumnado (Tabla 13). Mientras en el curso 2008-2009 destacan los alumnos que cursaron la opción de Tecnología, en el siguiente son los que cursaron Ciencias de la naturaleza y de la salud quienes sobresalen. 


\begin{tabular}{|c|c|c|c|c|c|c|c|c|c|c|}
\hline \multirow[t]{2}{*}{ Curso } & \multicolumn{2}{|c|}{$2005-2006$} & \multicolumn{2}{|c|}{ 2006-2007 } & \multicolumn{2}{|c|}{$2007-2008$} & \multicolumn{2}{|c|}{ 2008-2009 } & \multicolumn{2}{|c|}{$2009-2010$} \\
\hline & $t$ & $p$ & $t$ & $p$ & $t$ & $p$ & $F$ & $p$ & $F$ & $p$ \\
\hline Rendimiento 1 & 230 &, 835 & , 493 &, 624 & ,498 &, 620 & 3,055 &, 038 & 5,018 & 010 \\
\hline Media general & \multicolumn{2}{|c|}{4,482} & \multicolumn{2}{|c|}{4,470} & \multicolumn{2}{|c|}{4,735} & \multicolumn{2}{|c|}{4,920} & \multicolumn{2}{|c|}{4,270} \\
\hline
\end{tabular}

Tabla 13. Diferencias de rendimiento global con relación al tipo de Bachillerato cursado.

El número de convocatorias agotadas carece de de una relación clara con el nivel de rendimiento global (Tabla 14): el hecho de haber cursado esta materia anteriormente o no no implica diferencias sensibles de rendimiento en esta materia.

\begin{tabular}{|l|c|c|c|c|c|c|c|c|c|c|}
\hline \multirow{2}{*}{ Curso } & \multicolumn{2}{|c|}{$\mathbf{2 0 0 5 - 2 0 0 6}$} & \multicolumn{2}{c|}{$\mathbf{2 0 0 6 - 2 0 0 7}$} & $\mathbf{2 0 0 7 - 2 0 0 8}$ & $\mathbf{2 0 0 8 - 2 0 0 9}$ & \multicolumn{2}{c|}{$\mathbf{2 0 0 9 - 2 0 1 0}$} \\
\cline { 2 - 10 } & $F$ & $p$ & $F$ & $p$ & $F$ & $p$ & $F$ & $p$ & $F$ & $p$ \\
\hline Rendimiento 1 & 4,599 & $\mathbf{, 0 0 2}$ &, 876 &, 422 & 2,180 &, 100 &, 340 &, 713 &, 848 &, 432 \\
\hline Media general & \multicolumn{2}{|c|}{4,434} & \multicolumn{2}{|c|}{4,381} & \multicolumn{2}{|c|}{4,558} & \multicolumn{2}{|c|}{4,920} & \multicolumn{2}{|c|}{4,267} \\
\hline
\end{tabular}

Tabla 14. Diferencias de rendimiento global con relación al número de convocatorias agotadas.

El nivel medio de rendimiento de la parte teórica tiende a asemejarse al de la parte práctica (Tabla 15). Sin embargo, el índice de correlación entre ambas indica una relación significativa que es por término medio moderada.

\begin{tabular}{|c|c|c|c|c|c|}
\hline Curso & $\mathbf{2 0 0 5 - 2 0 0 6}$ & $\mathbf{2 0 0 6 - 2 0 0 7}$ & $\mathbf{2 0 0 7 - 2 0 0 8}$ & $\mathbf{2 0 0 8 - 2 0 0 9}$ & $\mathbf{2 0 0 9 - 2 0 1 0}$ \\
\hline Teoría 1 & 5,0108 & 4,3811 & 4,4286 & 4,7589 & 3,8702 \\
\hline Práctica 1 & 3,8565 & 4,4156 & 4,6865 & 5,0541 & 4,6063 \\
\hline$t$ & 4,144 &,- 106 &,- 834 &,- 779 & $-2,518$ \\
\hline$p$ & $\mathbf{, 0 0 0}$ &, 916 &, 407 &, 440 &, 014 \\
\hline$r_{x y}$ &, 478 &, 429 &, 607 & 469 &, 374 \\
\hline$p$ & $\mathbf{0 0 0}$ & $\mathbf{, 0 0 1}$ & $\mathbf{, 0 0 0}$ & $\mathbf{, 0 0 1}$ & $\mathbf{0 0 1}$ \\
\hline$d$ & $\mathbf{4 7 2}$ & $\mathbf{, 0 1 4}$ & $\mathbf{, 1 0 5}$ & $\mathbf{, 1 1 1}$ & $\mathbf{2 8 3}$ \\
\hline $\mathrm{N}$ & $\mathbf{7 7}$ & 61 & 63 & 49 & 79 \\
\hline
\end{tabular}

Tabla 15. Diferencias de rendimiento entre la parte teórica y la parte práctica.

En resumen, ni las características de entrada ni las de proceso permiten aseverar la existencia de una relación sensible con el rendimiento académico en la primera evaluación del curso. Ni siquiera el hecho de cursar esta materia en varias ocasiones implica resultados claramente diferenciados. A lo sumo se puede admitir que las dos partes en las que se divide el rendimiento están moderada y significativamente relacionadas entre sí y se asemejan en cuanto a su nivel medio. El tamaño del efecto que las separa oscila entre 0,014 y 0,472 dependiendo de la cohorte.

Fase III: Resultados de la evaluación de junio.

La evaluación de la parte teórica se lleva a cabo mediante una prueba de 60 ítems de elección múltiple que revisa la fundamentación teórico-práctica tanto de la investigación educativa como determinadas pruebas estadísticas básicas. El dominio 
de la parte práctica se evalúa mediante dos conjuntos de problemas similares en su diseño a los de febrero. Sobre cada uno de estos problemas se plantean varias preguntas orientadas a averiguar hasta qué punto el alumnado es capaz de aportar soluciones razonadas. La comparación de las medias obtenidas por los alumnos de las distintas opciones de aprendizaje arroja los mismos resultados que en febrero (Tabla 16). Los índices de correlación entre los dos aspectos también se comportan del mismo modo: son estadísticamente significativos pero moderados.

\begin{tabular}{|c|l|c|c|c|c|c|}
\hline \multicolumn{2}{|l|}{ Curso } & $\mathbf{2 0 0 5 - 2 0 0 6}$ & $\mathbf{2 0 0 6 - 2 0 0 7}$ & $\mathbf{2 0 0 7 - 2 0 0 8}$ & $\mathbf{2 0 0 8 - 2 0 0 9}$ & $\mathbf{2 0 0 9 - 2 0 1 0}$ \\
\hline \multirow{4}{*}{ Teoría 2 } & Media general & 4,307 & 4,376 & 4,220 & 4,341 & 3,636 \\
\cline { 2 - 7 } & $F$ &, 241 & 1,317 & 2,111 &, 442 &, 756 \\
\cline { 2 - 7 } & $p$ &, 868 &, 285 &, 114 &, 725 &, 475 \\
\cline { 2 - 7 } & $\eta^{2}$ &, 013 &, 107 &, 140 &, 042 &, 031 \\
\hline \multirow{5}{*}{ Práctica 2} & Media general & 6,060 & 5,984 & 5,617 & 5,488 & 4,730 \\
\cline { 2 - 7 } & $F$ & 2,203 &, 580 & 2,914 & 2,695 & 1,492 \\
\cline { 2 - 7 } & $p$ &, 098 &, 632 &, $\mathbf{0 4 6}$ &, 064 &, 235 \\
\cline { 2 - 7 } & $\eta^{2}$ &, 107 &, 050 &, $\mathbf{1 8 3}$ &, 212 &, 060 \\
\hline & $r_{x y}$ &, 451 &, 562 &, 480 &, 350 &, 454 \\
\cline { 2 - 7 } & $p$ & $\mathbf{0 0 0}$ & $\mathbf{, 0 0 0}$ & $\mathbf{, 0 0 1}$ & $\mathbf{, 0 4 3}$ & $\mathbf{, 0 0 1}$ \\
\cline { 2 - 7 } & $N$ & 36 & 43 & 34 & 50 \\
\hline
\end{tabular}

Tabla 16. Probabilidad asociada a las diferencias de rendimiento debidas a los métodos de aprendizaje.

Al igual que en febrero, el nivel de dificultad e interés manifestado por el alumnado al final del curso sigue sin aparecer como un factor relevante relacionado con el nivel de rendimiento global de junio. Al igual que en el caso anterior, en general el rendimiento se relaciona de manera débil o no significativa con el nivel de dificultad e interés (Tabla 17).

\begin{tabular}{|l|c|c|c|c|c|c|c|c|c|c|}
\hline \multirow{2}{*}{ Curso } & \multicolumn{2}{|c|}{$\mathbf{2 0 0 5 - 2 0 0 6}$} & \multicolumn{2}{|c|}{$\mathbf{2 0 0 6 - 2 0 0 7}$} & \multicolumn{2}{c|}{$\mathbf{2 0 0 7 - 2 0 0 8}$} & \multicolumn{2}{|c|}{$\mathbf{2 0 0 8 - 2 0 0 9}$} & \multicolumn{2}{c|}{$\mathbf{2 0 0 9 - 2 0 1 0}$} \\
\cline { 2 - 11 } & $r_{s}$ & $p$ & $r_{s}$ & $p$ & $r_{s}$ & $p$ & $r_{s}$ & $p$ & $r_{s}$ & $p$ \\
\hline Dificultad 2 &,- 193 &, 143 &,- 210 &, 242 &,- 132 &, 412 &,- 227 &, 236 &,- 453 &, 001 \\
\hline Interés 2 &, 045 &, 733 &,- 041 &, 820 &, 397 & $\mathbf{, 0 1 0}$ &, 316 &, 095 &, 426 &, 003 \\
\hline
\end{tabular}

Tabla 17. Relación entre el rendimiento global de junio y el nivel de dificultad e interés final.

El tipo de estudios cursados en Bachillerato sigue sin relacionarse de manera clara con el nivel de rendimiento global (Tabla 18).

\begin{tabular}{|c|c|c|c|c|c|c|c|c|c|c|}
\hline \multirow{2}{*}{ Curso } & \multicolumn{2}{|c|}{$\mathbf{2 0 0 5 - 2 0 0 6}$} & \multicolumn{2}{|c|}{$\mathbf{2 0 0 6 - 2 0 0 7}$} & $\mathbf{2 0 0 7 - 2 0 0 8}$ & $\mathbf{2 0 0 8 - 2 0 0 9}$ & \multicolumn{2}{|c|}{ 2009-2010 } \\
\cline { 2 - 10 } & $t$ & $p$ & $t$ & $p$ & $t$ & $p$ & $F$ & $p$ & $F$ & $p$ \\
\hline Rendimiento 2 &, 751 &, 456 & $-1,890$ &, 068 &, 252 &, 802 & 1,508 &, 233 & 1,097 &, 303 \\
\hline Media general & \multicolumn{2}{|c|}{5,462} & \multicolumn{2}{|c|}{4,932} & \multicolumn{2}{|c|}{4,978} & \multicolumn{2}{|c|}{4,938} & 4,6225 \\
\hline
\end{tabular}

Tabla 18. Diferencias de rendimiento global de junio debidas al tipo de bachillerato cursado. 
A diferencia de lo que ocurre en febrero, el nivel de rendimiento de la parte teórica es significativamente diferente al de la parte práctica (Tabla 19): la media de la parte práctica tiende a ser mayor que la de la parte teórica; el índice de correlación entre ambas sigue indicando una relación significativa que es por término medio moderada.

\begin{tabular}{|c|c|c|c|c|c|}
\hline Curso & $\mathbf{2 0 0 5 - 2 0 0 6}$ & $\mathbf{2 0 0 6 - 2 0 0 7}$ & $\mathbf{2 0 0 7 - 2 0 0 8}$ & $\mathbf{2 0 0 8 - 2 0 0 9}$ & $\mathbf{2 0 0 9 - 2 0 1 0}$ \\
\hline Teoría 2 & 4,3073 & 4,4114 & 4,2195 & 4,3415 & 3,6356 \\
\hline Práctica 2 & 6,0602 & 6,0778 & 5,6174 & 5,4882 & 4,7250 \\
\hline$t$ & $-5,922$ & $-4,349$ & $-3,333$ & $-2,517$ & $-2,757$ \\
\hline$p$ & $\mathbf{, 0 0 0}$ & $\mathbf{, 0 0 0}$ & $\mathbf{, 0 0 2}$ & $\mathbf{, 0 1 7}$ &, $\mathbf{0 0 8}$ \\
\hline$r_{x v}$ &, 451 &, 562 &, 480 &, 350 &, 454 \\
\hline$p$ & $\mathbf{, 0 0 0}$ & $\mathbf{, 0 0 0}$ & $\mathbf{, 0 0 1}$ & $\mathbf{, 0 4 3}$ & $\mathbf{0 0 1}$ \\
\hline$d$ & $\mathbf{, 7 7 1}$ & $\mathbf{, 7 2 5}$ & $\mathbf{, 5 0 8}$ & $\mathbf{4 3 2}$ & $\mathbf{3 9 0}$ \\
\hline $\mathrm{N}$ & 59 & 36 & 43 & 34 & 50 \\
\hline
\end{tabular}

Tabla 19. Diferencias de rendimiento entre la parte teórica y la parte práctica.

En resumen, como en febrero ni las características individuales de entrada y de salida ni las de proceso permiten aseverar la existencia de una relación sensible con el rendimiento académico en la última evaluación del curso. Las dos partes en las que se divide el rendimiento siguen moderada y significativamente relacionadas entre sí, siendo el nivel mayor en la parte práctica que en la teórica. El tamaño del efecto que las separa oscila entre 0,390 y 0,771 dependiendo de la cohorte. Este tamaño es mayor en junio que en febrero.

\section{Fase IV: Resultados globales del curso}

La puntuación final del curso es el equivalente de la media de las puntuaciones de aquellos alumnos que se presentaron tanto a la convocatoria de febrero como a la de junio.

Más del 65\% de los alumnos matriculados en este grupo se limitan a asistir a las tradicionales clases teóricas. En todo caso el método de aprendizaje elegido por cada alumno carece de un efecto diferenciador notable en el rendimiento en esta materia; se observan diferencias en sólo dos de los cinco cursos objeto de estudio. De hecho, la proporción de varianza explicada por los métodos tiende a estar por debajo del $20 \%$. Eso significa que otras variables no contempladas en este estudio explican una parte considerable del rendimiento (Tabla 20). Sin embargo, la comparación del rendimiento de aquellos alumnos que optaron por recurrir a alguna actividad complementaria para el estudio de esta materia con el de aquellos que decidieron acudir sólo a las clases teóricas sugiere que, si bien el efecto no es siempre estadísticamente significativo, al menos se orienta en el sentido de una mejora en todos los casos a favor de la realización actividades complementarias de aprendizaje. De hecho, el tamaño del efecto que se alcanza oscila entre 0,31 y 0,85 a lo largo de los cincos cursos (Tabla 21). 


\begin{tabular}{|c|c|c|c|c|c|}
\hline Curso & Método elegido & $N$ & Media & $F$ & $p$ \\
\hline \multirow{5}{*}{ 2005-2006 } & Sólo clase & 58 & 4,3083 & \multirow{5}{*}{2,618} & \multirow{5}{*}{$\begin{array}{c}, 057 \\
\eta^{2}=, 095\end{array}$} \\
\hline & Resolución de problemas & 9 & 5,4846 & & \\
\hline & Campus Virtual & 9 & 3,8085 & & \\
\hline & $\begin{array}{l}\text { Campus Virtual y Resolución de } \\
\text { problemas }\end{array}$ & 3 & 6,1967 & & \\
\hline & Total & 79 & 4,4571 & & \\
\hline \multirow{5}{*}{ 2006-2007 } & Sólo clase & 40 & 3,9046 & \multirow{5}{*}{4,866} & \multirow{5}{*}{$\eta^{2}=, 204$} \\
\hline & Resolución de problemas & 3 & 4,8050 & & \\
\hline & Campus Virtual & 12 & 4,8875 & & \\
\hline & $\begin{array}{l}\text { Campus Virtual y Resolución de } \\
\text { problemas }\end{array}$ & 6 & 6,5075 & & \\
\hline & Total & 61 & 4,3983 & & \\
\hline \multirow{6}{*}{ 2007-2008 } & Sólo clase & 24 & 3,6479 & \multirow{6}{*}{3,028} & \multirow{6}{*}{$\begin{array}{c}, 025 \\
\eta^{2}=, 173\end{array}$} \\
\hline & Campus Virtual & 15 & 4,9525 & & \\
\hline & Resolución de problemas & 12 & 5,5052 & & \\
\hline & $\begin{array}{l}\text { Campus Virtual y Resolución de } \\
\text { problemas }\end{array}$ & 1 & 1,5833 & & \\
\hline & Lecturas y ejercicios & 11 & 4,7687 & & \\
\hline & Total & 63 & 4,4752 & & \\
\hline \multirow{5}{*}{ 2008-2009 } & Sólo clase & 27 & 4,3444 & \multirow{5}{*}{1,227} & \multirow{5}{*}{$\begin{array}{l}310 \\
\eta^{2}=, 069\end{array}$} \\
\hline & Campus Virtual & 7 & 4,7175 & & \\
\hline & Resolución de problemas & 14 & 5,3955 & & \\
\hline & $\begin{array}{l}\text { Campus Virtual y Resolución de } \\
\text { problemas }\end{array}$ & 6 & 4,6550 & & \\
\hline & Total & 54 & 4,6998 & & \\
\hline \multirow{4}{*}{ 2009-2010 } & Sólo clase & 30 & 4,3545 & \multirow{4}{*}{1,397} & \multirow{4}{*}{$\eta^{2}=, 067$} \\
\hline & Campus Virtual & 6 & 4,4171 & & \\
\hline & Resolución de problemas & 6 & 5,6146 & & \\
\hline & Total & 42 & 4,5435 & & \\
\hline
\end{tabular}

Tabla 20. Probabilidad asociada a las diferencias de rendimiento final entre alumnos en función del método elegido.

\begin{tabular}{|c|c|c|c|c|c|}
\hline Curso & Método elegido & $N$ & Media & $t$ & $p$ \\
\hline \multirow{2}{*}{ 2005-2006 } & Sólo clase & 58 & 4,3083 & \multirow{2}{*}{1,233} & \multirow{2}{*}{$\begin{array}{c}, 221 \\
d=0,31\end{array}$} \\
\hline & Actividades complementarias & 21 & 4,8680 & & \\
\hline \multirow{2}{*}{ 2006-2007 } & Sólo clase & 40 & 3,9046 & \multirow{2}{*}{3,164} & \multirow{2}{*}{$\begin{array}{c}, 002 \\
d=0,85\end{array}$} \\
\hline & Actividades complementarias & 21 & 5,3386 & & \\
\hline \multirow{2}{*}{ 2007-2008 } & Sólo clase & 24 & 3,6479 & \multirow{2}{*}{2,738} & \multirow{2}{*}{$\begin{array}{c}, 008 \\
d=0,71\end{array}$} \\
\hline & Actividades complementarias & 39 & 4,9843 & & \\
\hline \multirow{2}{*}{ 2008-2009 } & Sólo clase & 27 & 4,3444 & \multirow{2}{*}{1,581} & \multirow{2}{*}{$\begin{array}{c}, 120 \\
d=0,43\end{array}$} \\
\hline & Actividades complementarias & 27 & 5,0552 & & \\
\hline \multirow{2}{*}{ 2009-2010 } & Sólo clase & 30 & 4,3545 & \multirow{2}{*}{1,134} & \multirow{2}{*}{$\begin{array}{c}, 264 \\
d=0,39\end{array}$} \\
\hline & Actividades complementarias & 12 & 5,0159 & & \\
\hline
\end{tabular}

Tabla 21. Probabilidad asociada a las diferencias de rendimiento final entre alumnos con y sin actividades complementarias de aprendizaje. 
El tipo de estudios cursados en Bachillerato implica diferencias más bien poco relevantes en el nivel de rendimiento final en esta materia. Sin embargo, llama la atención el hecho de que los alumnos procedentes de Letras o que se consideran como tales tengan invariablemente una media menor aunque la diferencia con respecto a los de Ciencias sea muy pequeña (Tabla 22).

\begin{tabular}{|c|c|c|c|c|c|}
\hline Curso & Grupo & $N$ & Media & $t-F$ & $p$ \\
\hline \multirow{2}{*}{ 2005-2006 } & Ciencias & 14 & 4,6946 & \multirow{2}{*}{$t=, 425$} & \multirow{2}{*}{672} \\
\hline & Letras & 59 & 4,4668 & & \\
\hline \multirow{2}{*}{ 2006-2007 } & Ciencias & 12 & 4,7471 & \multirow{2}{*}{$t=, 718$} & \multirow{2}{*}{,476 } \\
\hline & Letras & 43 & 4,3217 & & \\
\hline \multirow{2}{*}{ 2007-2008 } & Ciencias & 14 & 4,7420 & \multirow{2}{*}{$t=, 358$} & \multirow{2}{*}{, 722} \\
\hline & Letras & 40 & 4,5220 & & \\
\hline \multirow{4}{*}{ 2008-2009 } & Artes & 1 & 7,7575 & \multirow{4}{*}{$F=2,353$} & \multirow{4}{*}{, 084} \\
\hline & $\begin{array}{l}\text { Ciencias de la naturaleza y de la } \\
\text { salud }\end{array}$ & 8 & 5,5947 & & \\
\hline & Humanidades y Ciencias Sociales & 42 & 4,4507 & & \\
\hline & Tecnología & 2 & 5,1050 & & \\
\hline \multirow[t]{2}{*}{ 2009-2010 } & $\begin{array}{l}\text { Ciencias de la naturaleza y de la } \\
\text { salud }\end{array}$ & 10 & 5,0722 & \multirow[t]{2}{*}{$F=1,097$} & \multirow[t]{2}{*}{,303 } \\
\hline & Humanidades y Ciencias Sociales & 23 & 4,4269 & & \\
\hline
\end{tabular}

Tabla 22. Probabilidad asociada a las diferencias de rendimiento final entre los alumnos de Letras y de Ciencias.

En el nivel de interés se producen pequeñas variaciones que sugieren un pequeño incremento entre el principio y el final del curso. En cuanto al nivel de dificultad los cambios parecen más sensibles y se orientan hacia una cierta disminución en algunos casos significativa (Tabla 23) en aquellos alumnos que logran terminar el curso.

\begin{tabular}{|c|c|c|c|c|c|c|c|c|c|c|c|}
\hline \multirow{2}{*}{\multicolumn{2}{|c|}{ Curso }} & \multicolumn{2}{|c|}{$2005-2006$} & \multicolumn{2}{|c|}{ 2006-2007 } & \multicolumn{2}{|c|}{$2007-2008$} & \multicolumn{2}{|c|}{ 2008-2009 } & \multicolumn{2}{|c|}{ 2009-2010 } \\
\hline & & $Z$ & $p$ & $Z$ & $p$ & $Z$ & $p$ & $Z$ & $p$ & $Z$ & $P$ \\
\hline \multicolumn{2}{|c|}{ Dificultad } & $-2,583$ & ,010 & $-1,998$ & ,046 & $-3,757$ & ,000 &,- 748 &, 454 & $-3,899$ & ,000 \\
\hline \multirow{2}{*}{ Media } & Inicial & \multicolumn{2}{|c|}{7,928} & \multicolumn{2}{|c|}{8,173} & \multicolumn{2}{|c|}{8,044} & \multicolumn{2}{|c|}{7,460} & \multicolumn{2}{|c|}{6,420} \\
\hline & Final & \multicolumn{2}{|c|}{7,419} & \multicolumn{2}{|c|}{7,764} & \multicolumn{2}{|c|}{7,168} & \multicolumn{2}{|c|}{7,448} & \multicolumn{2}{|c|}{7,883} \\
\hline \multicolumn{2}{|c|}{ Interés } & $-1,022$ &, 307 & $-1,660$ & ,097 & $-1,611$ &, 107 &,- 911 &, 362 & $-1,525$ &, 127 \\
\hline \multirow{2}{*}{ Media } & Inicial & \multicolumn{2}{|c|}{5,625} & \multicolumn{2}{|c|}{5,525} & \multicolumn{2}{|c|}{5,589} & \multicolumn{2}{|c|}{6,370} & \multicolumn{2}{|c|}{5,840} \\
\hline & Final & \multicolumn{2}{|c|}{5,648} & \multicolumn{2}{|c|}{6,133} & \multicolumn{2}{|c|}{6,122} & \multicolumn{2}{|c|}{7,034} & \multicolumn{2}{|c|}{6,243} \\
\hline
\end{tabular}

Tabla 23. Probabilidad asociada a las diferencias de percepción entre el final y el principio del curso.

El rendimiento en la convocatoria de junio está significativamente relacionado con el de febrero; sin embargo, el índice de correlación es relativamente bajo (Tabla 24); apenas supera 0,540 por término medio, lo que supone menos del $30 \%$ de varianza común. Por otra parte, la media de rendimiento de aquellos alumnos que logran terminar el curso tiende a ser similar a la obtenida en la convocatoria de febrero. Sin embargo, el tamaño del efecto que las separa varía bastante entre las cohortes: oscila 
entre 0,033 y 0,650 pero en sentido opuesto: en los últimos tres cursos el rendimiento de junio está sensiblemente por debajo del de febrero. De hecho, más del $58 \%$ de los alumnos que han realizado ambos exámenes están en esta situación.

\begin{tabular}{|l|l|c|c|c|c|c|}
\hline \multicolumn{2}{|c|}{ Curso } & $\mathbf{2 0 0 5 - 2 0 0 6}$ & $\mathbf{2 0 0 6 - 2 0 0 7}$ & $\mathbf{2 0 0 7 - 2 0 0 8}$ & $\mathbf{2 0 0 8 - 2 0 0 9}$ & $\mathbf{2 0 0 9 - 2 0 1 0}$ \\
\hline Febrero & Media general & 5,1469 & 4,9270 & 5,1597 & 5,8224 & 4,7740 \\
\hline Junio & Media general & 5,2083 & 5,5229 & 4,9185 & 4,8255 & 4,3129 \\
\hline & $r_{x y}$ &, 401 &, 446 &, 763 &, 542 &, 558 \\
\cline { 2 - 7 } & $p$ &, $\mathbf{0 0 2}$ &, $\mathbf{0 0 9}$ &, $\mathbf{0 0 0}$ & $\mathbf{, 0 0 2}$ &, $\mathbf{0 0 0}$ \\
\cline { 2 - 7 } & $t$ &, 252 & 2,075 & $-1,160$ & $-3,499$ & $-1,616$ \\
\cline { 2 - 7 } & $p$ &, 802 &, $\mathbf{0 4 6}$ &, 253 &, $\mathbf{0 0 2}$ &, 114 \\
\cline { 2 - 7 } & $d$ &, 033 &, $\mathbf{3 6 1}$ &, 177 & $\mathbf{6 5 0}$ &, 249 \\
\cline { 2 - 7 } & $N$ & 57 & 33 & 43 & 29 & 42 \\
\hline
\end{tabular}

Tabla 24. Probabilidad asociada a las diferencias de rendimiento entre febrero y junio.

El número de convocatorias agotadas no aparece como un factor diferenciador potente con relación al nivel de rendimiento final en esta materia- (Tabla 25). Los tamaños del efecto indican que las convocatorias agotadas explican una proporción prácticamente insiginificante de la varianza total. Sin embargo, llama la atención una llamativa observación: aunque las diferencias no sean significativas, los alumnos que repiten por primera vez tienden a obtener un rendimiento inferior al de los alumnos que cursan esta asignatura por primera vez. La segunda y tercera repeticiones suelen terminar con una mejora más notable.

\begin{tabular}{|c|c|c|c|c|c|}
\hline Curso & Convocatorias agotadas & $N$ & Media & $F$ & $P$ \\
\hline \multirow{6}{*}{ 2005-2006 } & Ninguna & 47 & 4,1841 & \multirow{6}{*}{3,683} & \multirow{6}{*}{$\begin{array}{c}, 009 \\
\eta^{2}=, 166\end{array}$} \\
\hline & Una & 9 & 3,5320 & & \\
\hline & Dos & 14 & 4,9389 & & \\
\hline & Tres & 8 & 5,8249 & & \\
\hline & Cuatro & 1 & 7,9208 & & \\
\hline & Total & 79 & 4,4571 & & \\
\hline \multirow{4}{*}{ 2006-2007 } & Ninguna & 53 & 4,3785 & \multirow{4}{*}{1,703} & \multirow{4}{*}{$\begin{array}{c}, 191 \\
\eta^{2}=, 056\end{array}$} \\
\hline & Una & 7 & 4,0929 & & \\
\hline & Dos & 1 & 7,5850 & & \\
\hline & Total & 61 & 4,3983 & & \\
\hline \multirow{5}{*}{ 2007-2008 } & Ninguna & 43 & 4,6150 & \multirow{5}{*}{2,049} & \multirow{5}{*}{$\begin{array}{c}, 117 \\
\eta^{2}=, 094\end{array}$} \\
\hline & Una & 12 & 3,9283 & & \\
\hline & Dos & 6 & 5,4203 & & \\
\hline & Tres & 2 & 1,9167 & & \\
\hline & Total & 63 & 4,4752 & & \\
\hline \multirow{5}{*}{ 2008-2009 } & Ninguna & 42 & 4,4561 & \multirow{5}{*}{1,723} & \multirow{5}{*}{$\begin{array}{c}, 174 \\
\eta^{2}=, 094\end{array}$} \\
\hline & Una & 4 & 5,6150 & & \\
\hline & Dos & 7 & 5,3025 & & \\
\hline & Tres & 1 & 7,0550 & & \\
\hline & Total & 54 & 4,6998 & & \\
\hline 2009-2010 & Ninguna & 40 & 4,4855 &, 835 & 441 \\
\hline
\end{tabular}




\begin{tabular}{|l|l|c|c|c|c|}
\hline & Dos & 1 & 4,6736 & & $\eta^{2}=, 041$ \\
\cline { 2 - 4 } & Tres & 1 & 6,7333 & & \\
\cline { 2 - 4 } & Total & 42 & 4,5435 & & \\
\hline
\end{tabular}

Tabla 25. Diferencias de rendimiento final debidas al número de convocatorias agotadas.

Las diferencias de rendimiento entre las cohortes no resultan estadísticamente significativas (Tabla 26). Además, los tamaños del efecto advierten que las cohortes no explican casi nada de la varianza total.

\begin{tabular}{|c|c|c|c|c|c|c|c|c|c|c|c|c|}
\hline & \multicolumn{4}{|c|}{ Febrero } & \multicolumn{4}{|c|}{ Junio } & & \multicolumn{3}{|c|}{ Final } \\
\hline & $\mathbf{N}$ & Media & $F$ & $p$ & $\mathbf{N}$ & Media & $F$ & $p$ & $\mathbf{N}$ & Media & $F$ & $P$ \\
\hline $\begin{array}{c}2005- \\
2006\end{array}$ & 77 & 4,4337 & \multirow{5}{*}{1,137} & \multirow{5}{*}{,339 } & 59 & 5,1837 & \multirow{5}{*}{2,245} & \multirow{5}{*}{,065 } & 79 & 4,4571 & \multirow{5}{*}{, 234} & \multirow{5}{*}{919} \\
\hline $\begin{array}{c}2006- \\
2007\end{array}$ & 61 & 4,3810 & & & 36 & 5,2154 & & & 61 & 4,3983 & & \\
\hline $\begin{array}{c}2007- \\
2008\end{array}$ & 63 & 4,5575 & & & 43 & 4,9185 & & & 63 & 4,4752 & & \\
\hline $\begin{array}{c}2008- \\
2009\end{array}$ & 49 & 4,9200 & & & 34 & 4,9149 & & & 54 & 4,6998 & & \\
\hline $\begin{array}{c}2009- \\
2010\end{array}$ & 79 & 4,2668 & & & 50 & 4,1803 & & & 42 & 4,5434 & & \\
\hline Total & 329 & 4,4800 & $\eta^{2}=$ & 014 & 222 & 4,8703 & $\eta^{2}=$ & 058 & 299 & 4,5049 & $\eta^{2}=$ & 003 \\
\hline
\end{tabular}

Tabla 26. Diferencias de rendimiento entre cohortes.

En resumen, las características individuales de entrada y de proceso no permiten aseverar la existencia de una relación sensible con el rendimiento académico del curso. De hecho, los métodos elegidos por los alumnos carecen de un efecto diferenciador claro. Sin embargo, la realización de actividades de aprendizaje complementarias produce efectos positivos.

\section{Discusión}

Globalmente los alumnos de los cinco cursos muestran relativamente poca variación tanto en sus características personales como en sus resultados parciales y finales.

Un porcentaje próximo al $60 \%$ se matricula y no se presenta a examen o abandona tras suspender el primero de los dos exámenes del curso (McInnis et al., 2000). Suelen iniciar el curso pensando que la materia es difícil o muy difícil y además no resulta excesivamente atrayente (McKenzie \& Schweitzer, 2001). Esta situación se podría deber a la imagen indudablemente negativa que las cohortes precedentes propagan, tras asistir a no más del $20 \%$ de las clases teóricas o realizar alguna actividad de aprendizaje que les desanima porque la consideran complicada. Al final del curso esta percepción varía relativamente poco en términos absolutos. Sin embargo, disminuye significativamente la percepción del nivel de dificultad en ese $40 \%$ de alumnos que esfuerzan por trabajar a lo largo del curso. Aparentemente a 
estos alumnos las distintas actividades de aprendizaje que se les ofrece les permiten revisar positivamente su percepción de la materia. Lo mismo ocurre con el nivel de interés pero en menor medida. Ahora bien, es probable que ese $60 \%$ de alumnos que no asisten a clase y/o no se presentan a examen siga teniendo y propagando una percepción realmente negativa de esta materia.

Los alumnos suelen llegar con un nivel de alfabetización estadística más bien pobre: sería más correcto decir que sus conocimientos previos carecen del nivel esperado si se tiene en cuenta lo que los programas preuniversitarios oficiales prevén. Da la impresión de que los alumnos llegan a las aulas universitarias con el nivel de $4^{\circ}$ ESO. Éste es un nivel que no permite predecir de modo fiable el rendimiento en la universidad. Tampoco es fiable el resultado obtenido al medir los conocimientos previos partiendo de los programas de Bachillerato. Llama la atención la discrepancia que hay entre lo que los alumnos aprenden en los cursos de Bachillerato y lo que los profesores universitarios esperan como preparación adquirida. De hecho, la relación entre el nivel de conocimientos previos y el del rendimiento del primer examen así lo sugiere. Es probable que esta situación sea consecuencia de la escasa importancia que se otorga a la estadística en el bagaje académico del futuro universitario, como sugiere Estrada (2002).

La falta de hábitos de trabajo intelectual se refleja en el modo de reaccionar ante la oferta de estrategias de aprendizaje de esta materia: en torno al 67,5\% de estos alumnos prefiere asistir solamente a las tradicionales clases teóricas o ni siquiera eso. Para la mayoría la toma de apuntes sigue siendo la principal vía de adquisición de conocimientos, no necesariamente de habilidades. El restante tercio intenta compaginar la asistencia a clase con otras técnicas obteniendo resultados que evidencian cierta mejora, aunque sea relativamente limitada. Ahora bien, el hecho de que la compaginación de estrategias no arroje resultados claramente mejores se puede deber a esa falta de hábitos de trabajo intelectual. Así, resulta llamativo el hecho de que algunos alumnos que se deciden por el sistema de apoyo a través de la plataforma WebCt entreguen ejercicios copiados unos de otros, incluidos los errores y las faltas de ortografía. La asignación de ejercicios personalizados reduce la tentación de copiar pero la utilización repetitiva de ciertos formulismos o de razonamientos ilógicos sigue denotando un insuficiente esfuerzo personal por dominar la materia más allá de ciertos aspectos en buena medida mecánicos. Este mismo comportamiento también se observa entre aquellos alumnos que deciden acudir a realizar ejercicios de solución de problemas: en vez de traerlos resueltos o al menos haberlo intentado, se presentan para tomar apuntes de los ejercicios resueltos paso a paso por el monitor. El insistente rechazo a realizar ejercicios fuera del aula es otra señal que muestra esta falta de entrenamiento para un trabajo personal. Sin embargo, hay que reconocer que la realización de cualquier actividad complementaria al trabajo del aula tiene el potencial de incrementar la probabilidad de mejorar el nivel de rendimiento.

El tipo de estudios cursados en Bachillerato no es un predictor potente y fiable del rendimiento que un alumno puede lograr en esta materia. A lo sumo se puede sugerir que los alumnos que proceden del área de Ciencias probablemente rindan escasamente mejor que los de Letras. Ésta es una situación que es en cierto modo insólita. Si los 
alumnos que cursan estudios relacionados con Ciencias reciben más contenidos de orientación lógico-matemática deberían rendir de modo claramente superior a los de Letras en esta materia. Cabe pensar que el nivel de dominio de los contenidos lógicomatemáticos alcanzado por estos alumnos de Ciencias es lo suficientemente bajo como para no distinguirse del logrado por los de Letras.

El rendimiento logrado por estos alumnos está muy débilmente relacionado con la percepción que tienen del nivel de dificultad e interés de esta materia. Tampoco se observan diferencias notables en función del número de convocatorias agotadas. A este respecto resulta interesante destacar una cierta anomalía. En condiciones normales lo lógico sería esperar que los alumnos que ya cursaron esta materia rindieran por encima de aquellos que se matriculan por primera vez. Curiosamente, aunque las diferencias no sean significativas los alumnos que repiten por primera vez rinden por debajo de todos sus compañeros. Esta situación podría deberse a una excesiva confianza de los repetidores primerizos en su capacidad para aprobar sin ni siquiera asistir a clase. A pesar de las advertencias se matriculan a la vez en esta materia y en cursos avanzados cuyos horarios no pueden evitar el solapamiento. Confiando en que con lo aprendido el curso anterior será suficiente se dan cuenta del error que han cometido cuando se sientan a realizar los exámenes: tratándose de una materia que exige más razonamiento que memorización, no son capaces de prepararla por su cuenta debido a su falta de hábitos de trabajo intelectual. Con frecuencia la solución consiste en abandonar esta materia hasta terminar todas las demás.

La escasa relación observada entre la parte teórica y la parte práctica revela cierta dificultad que tienen los alumnos para aplicar sus limitados conocimientos teóricos para resolver problemas prácticos relativos a la investigación en el entorno educativo. Esto a su vez deja planteada la duda de si son capaces de leer y entender los resultados publicados en determinadas revistas de investigación educativa, especialmente cuando se utiliza un vocabulario técnico altamente especializado. No obstante, la moderada relación que se observa entre ambas partes parece mejorar pero de manera poco significativa a lo largo del curso. Además, los alumnos manifiestan una leve mejora en su capacidad para rendir en la parte práctica. Ahora bien, permanece el problema de la distancia entre los contenidos teóricos y la realidad.

La ausencia de diferencias de rendimiento en la parte teórica en ambas convocatorias entre las distintas opciones de aprendizaje de esta materia sugiere que el efecto del profesor es constante entre cursos y a lo largo de cada curso. Las ocasionales diferencias que se observan en la parte práctica pudieran deberse al mayor o menor impacto de los monitores debido probablemente a su amplia o reducida experiencia en este tipo de tareas.

\section{A modo de conclusión}

Las características de entrada y de proceso están débilmente relacionadas con los resultados que alcanzan los alumnos. No se observan efectos interactivos significativos y constantes entre las estrategias de aprendizaje adoptadas por los alumnos, el tipo de estudios cursados en Bachillerato o el número de convocatorias 
agotadas. Las diferencias de rendimiento parecen mediadas por un conjunto de variables externas a la propia materia y no contraladas: además de la ansiedad cuyos efectos ya se conocen posiblemente habría que incluir los hábitos de estudios. El rendimiento insatisfactorio que describe este estudio se asemeja al que los investigadores Arum y Roksa (2010) exponen en su libro "Academically Adrift. Limited Learning on College Campuses": tras estudiar a 2.300 universitarios de 24 universidades concluyen que la mayoría de los graduados no aprende prácticamente nada.

Siendo un estudio de campo los resultados reseñados así como los comentarios que acompañan han de tomarse con la debida cautela. De hecho, no se ha pretendido aleatorizar ni muestrear sino tomar un contexto real, con todas sus condiciones algunas conocidas y otras desconocidas y estudiar los efectos de unas mejoras introducidas bajo la forma de actividades complementarias propuestas a los alumnos. La aparente carencia de efectos potentes de estas actividades tomadas por separado sugiere la necesidad de seguir buscando, sobre todo si los resultados muestran que en su conjunto son una aportación positiva frente a las simples clases teóricas. La mejora de las estrategias de control de las variables extrañas es sin duda una necesidad para seguir avanzando.

\section{Referencias bibliográficas}

ARUM, R. \& RoKSA J. (2010). Academically Adrift. Limited Learning on College Campuses. Chicago: The University of Chicago Press Books.

BAZÁN, J. L. Y APARICIO, A. S. (2006). Las actitudes hacia la Matemática-Estadística dentro de un modelo de aprendizaje, Revista Semestral del Departamento de Educación, XV, 28.

BIEHLER, R. (2008). From statistical literacy to fundamental ideas in mathematics: how can we bridge the tension in order to support teachers of statistics, en C. Batanero, G. Burril, C. Reading \& A. Rossman (Eds.), Joint ICMI/IASE Study: Teaching Statistics in School Mathematics. Challenge for Teaching and Teacher Education. Proceedings of the ICMI Study 18 and 2008 IASE Round Table Conference. Monterrey, Mexico: International Commission on Mathematical Instruction and International Association for Statistical Education.

Blanco Blanco, A. (2008). Una revisión crítica de la investigación sobre las actitudes de los estudiantes universitarios hacia la Estadística. Revista Complutense de Educación 19, 2, 311-330.

BRUINSMA, M. (2003). Effectiveness of higher education. Factors that determine outcomes of university education. Veenendaal: Universal Press.

CABAllero, A. Y Blanco, L. J. (2007). Las actitudes y emociones ante las Matemáticas de los estudiantes para Maestros de la Facultad de Educación de la Universidad de Extremadura. Comunicación presentada en el Grupo de Trabajo "Conocimiento y desarrollo profesional del profesor", en el XI SEIEM. Simposio de Investigación y Educación Matemática, celebrado en la Universidad de La Laguna los días 4 al 7 de Septiembre de 2007. 
CARMONA MÁRQUEZ, J. (2004). Una revisión de las evidencias de fiabilidad y validez de los cuestionarios de actitudes y ansiedad hacia la estadística..Statistics Education Research Journal 3(1), 5-28.

Cuevas Acosta, J. H. E IBÁÑEZ Bernal, C. (2008). Estándares en educación estadística: Necesidad de conocer la base teórica y empírica que los sustentan. Unión 15, 33- 45.

Darias Morales, E. J. (2000). Escala de actitudes hacia la estadística. Psicothema, 12, Supl. no 2, 175-178.

EPPleR, M. A. \& HARJU, B. L. (1997). Achievement motivation goals in relation to academic performance in traditional and non-traditional college students. Research in Higher Education, 38 (5), 557-573.

ESTRADA, A. (2001). Evaluación de actitudes hacia la estadística, en J. M. Cardeñoso et al., (Eds.). Actas de las Jornadas de Investigación en el aula de Matemáticas. Atención a la diversidad, 157-162. Departamento de Didáctica de la Matemática. Universidad de Granada.

ESTRADA, A. (2002). Análisis de las actitudes y conocimientos estadísticos elementales en la formación del profesorado. Universidad Autónoma de Barcelona. Tesis doctoral.

ESTRADA, A.; BATANERO, C. Y FORTUNY, J. M. (2004). Un estudio comparado de las actitudes hacia la estadística en profesores en formación y en ejercicio. Enseñanza de las Ciencias, 22(2), 263-274.

ESTRADA, A. Y BATANERO, C. (2008). Explaining teachers' attitudes towards statistics en C. Batanero, G. Burrill, C. Reading y A. Rossman (Eds.), Joint ICMI/IASE Study: Teaching Statistics in School Mathematics. Challenges for Teaching and Teacher Education. Proceedings of the ICMI Study 18 and 2008 IASE Round Table Conference. Monterrey, Mexico: International Commission on Mathematical Instruction and International Association for Statistical Education.

HAtiva, N. \& BiREnBAUM, M. (2000). Who prefers what? Disciplinary differences in students' preferred approaches to teaching and learning styles. Research in Higher Education, 41 (2), 209-236.

INCE (2001). Fallos y dificultades de los alumnos en la prueba de Matemáticas. Madrid: INCE.

LIAW, S. (2008). Investigating students' perceived satisfaction, behavioral intention, and effectiveness of e-learning: A case study of the Blackboard system. Computers \& Education, 51(2), 864-873.

LizZIO, A., WiLsON, K. \& Simons, R. (2002). University students' perceptions of the learning environment and academic outcomes: implications for theory and practice. Studies in Higher Education, 27 (1), 27-53.

LLINARES, S (2008). Agendas de investigación en Educación Matemática en España. Una aproximación desde "ISI-web of knowledge" y ERIH., en R. Luengo;, B. Gómez, M. y L. Camacho Blanco (Eds.) (2008). Investigación en educación Matemática XII, 25-54. Badajoz: SEIEM. 
MAFOKOZI, J. (2006). An introductory course to statistical concepts, theory and designs in educational research methods: a combination of strengths and weaknesses. ICOTS-7 Conference Proceedings, 1-4. Salvador de Bahía (Brasil).

Mcinnis, C., Hartley, R., Polesel, J. \& Teese, R. (2000). Non-completion in vocational education and training and higher education. Canberra, Australia: DETYA.

MCKenzie, K. \& Schweitzer, R. (2001). Who succeeds at university? Factors predicting academic performance in first year Australian university students. Higher Education Research \& Development, 20 (1), 21-33.

MondéJar Jiménez, J., VArgas VArgas, M. Y BAyot Mestre, A. (2008). Medición de la actitud hacia la estadística. Influencia de los procesos de estudio. Revista Electrónica de Investigación Psicoeducativa 16, 6(3), 729-748.

MuÑoz SAN RoQUe, I. (2002). Actitudes hacia la estadística y su relación con otras variables en alumnos universitarios del área de las Ciencias Sociales. Universidad Comillas. Tesis doctoral.

NORTES CHECA, A. (1998). Estadística y probabilidad una propuesta didáctica para la enseñanza secundaria. Revista Interuniversitaria de Formación del Profesorado 32, 59-72.

PeÑaloza FigueroA, J. L. \& VArgas Pérez, C. G. (2006). ¿Qué debe cambiar en el aprendizaje de la estadística en las ciencias del comportamiento? XIV Jornadas ASEPUMA- II Encuentro Internacional. Badajoz, España.

Pérez-Tyteca, P., Castro, E., Segovia, I., Castro, E., Fernández, F. y Cano, F. (2009). El papel de la ansiedad matemática en el paso de la educación secundaria a la educación universitaria. $P N A, 4(1), 23-35$.

PIKE, G. R. \& SAUPE, J. L. (2002). Does high school matter? An analysis of three methods of predicting first-year grades. Research in Higher Education, 43 (2), 187-207.

SÁNCHEZ HueTE, J. C. (2004). ¿Por qué las matemáticas básicas cambian? Claves para entender las renovaciones curriculares. Educación y futuro: revista de investigación aplicada y experiencias educativas, 1576-5199, 11, 161-170.

SIERRA VÁZQUEZ, M. (1989). La enseñanza de la matemática en la E.G.B. en España (1970-1985). Revista de Pedagogía de la Universidad de Salamanca, 2, 69-74.

\section{Fuentes digitales}

BATANERO, C. (2007) ¿Hacia dónde va la educación estadística? [Formato html] http://www.ugr.es/ batanero/ARTICULOS/BLAIX.htm (Consultado 6 de febrero de 2010).

BATANERO, C. (2009) Educación estadística en los niveles no universitarios. Oportunidades y desafíos actuales [Formato pdf] http://www.sgapeio.es/actividades/congresos/ourense 2009/resumenes/Batanerosgapeio2009.pdf (Consultado 6 de febrero de 2010) 
REAL SOCIEDAD MATEMÁTICA ESPAÑOLA (2008). Sobre el Informe PISA 2006 de Matemáticas: tres retos y una petición para los próximos años [Formato pdf] http://www.rsme.es/comis/educ/PISA06-RSME.pdf (Consultado 6 de junio de 2010)

RICO, L. Y SIERRA, M. (1997) Historia del Currículo de Matemáticas en España para Educación Secundaria (1970-1990)

[Formato pdf] http://cumbia.ath.cx:591/pna/Archivos/RicoL97-110.PDF (Consultado 15 de mayo de 2010)

\section{Correspondencia con el autor}

Joseph Mafokozi

Dpto. Métodos de Investigación y Diagnóstico en Educación

Universidad Complutense de Madrid

e-mail: mafjos@edu.ucm.es 